\title{
About a Class of Positive Hybrid Dynamic Linear Systems and an Associate Extended Kalman-Yakubovich-Popov Lemma
}

\author{
M. De la Sen \\ Instituto de Investigación y Desarrollo de Procesos (IIDP), Facultad de Ciencia y Tecnologia, Universidad del País Vasco, \\ Leioa (Bizkaia), P.O. Box 644 de Bilbao, 48080 Bilbao, Spain
}

Correspondence should be addressed to M. De la Sen; manuel.delasen@ehu.eus

Received 6 September 2017; Accepted 12 November 2017; Published 13 December 2017

Academic Editor: Delfim F. M. Torres

Copyright (C) 2017 M. De la Sen. This is an open access article distributed under the Creative Commons Attribution License, which permits unrestricted use, distribution, and reproduction in any medium, provided the original work is properly cited.

\begin{abstract}
This paper formulates an "ad hoc" robust version under parametrical disturbances of the discrete version of the KalmanYakubovich-Popov Lemma for a class of positive hybrid dynamic linear systems which consist of a continuous-time system coupled with a discrete-time or a digital one. An extended discrete system, whose state vector contains both the digital one and the discretization of the continuous-time one at sampling instants, is a key analysis element in the formulation. The hyperstability and asymptotic hyperstability properties of the studied class of positive hybrid systems under feedback from any member of a nonlinear (and, eventually, time-varying) class of controllers, which satisfies a Popov's-type inequality, are also investigated as linked to the positive realness of the associated transfer matrices.
\end{abstract}

\section{Introduction}

Continuous-time and discrete-time positive systems have been studied in detail in recent years [1-10]. In particular, if both the state and output possess such a property, the positivity is said to be internal or, simply, the system is positive. If the output possesses such a property, the system is said to be externally positive. Therefore, positive systems are intrinsically interesting to describe some problems like Markov chains, queuing problems, certain distillation columns, and biological and other physical compartmental problems where populations or concentrations cannot be negative $[2,3]$. A related property is that time-invariant dynamic linear systems which are externally positive, while they have positive real or strictly positive real transfer matrices, are, in addition, hyperstable or asymptotically hyperstable, that is, globally Lyapunov stable for any nonlinear and/or time-varying feedback device satisfying a Popov's-type inequality for all time [11, 12]. Such a property of asymptotic hyperstability generalizes that of absolute stability [13-15], which generalizes the most basic concept of stability of dynamic systems. See, for instance, [13, 14, 16-29] and references therein. The hyperstability property, which has a frequency-based physical interpretation in terms of positive realness of the transfer function of a feed-forward linear block, is also related to external positivity of the inputoutput relation rather than to (internal) positivity of the state-trajectory solution what is equivalent to positivity of the instantaneous input-output power and the input-output energy $[2,3,13,15,30]$. It is well known that closed-loop hyperstability is, by nature, a powerful version of closedloop stability since it refers to the stability of an hyperstable linear feed-forward plant (in the sense of positive realness of the associate transfer matrix) under a wide class of feedback controllers applied. The above important properties make very attractive potential research issues for kind of more complex dynamic systems with applied projection including those lying in the class of continuous/digital hybrid systems. On the other hand, the class of hybrid systems consisting of continuous-time and discrete-time (or digital) systems are of an increasing interest since many existing industrial installations combine both kinds of systems. An elementary well-known case is when a discrete-time controller is used for a continuous-time plant. Another case is related to teleoperation systems where certain variables evolve in a discrete-time or digital fashion. A background literature and related relevant results are given in $[1,7,11,16,17,26,31,32]$ and some of the references therein. The objective of this paper is to address appropriate versions of the Kalman-YakubovichPopov Lemma (KYP-Lemma) for a class of hybrid systems 
consisting of coupled linear continuous-time and digital dynamic subsystems, firstly proposed in [31], provided that they are, furthermore, positive [7], in the sense that, for any initial condition and any admissible controls both with nonnegative components, all the components of the state and output trajectory solutions are nonnegative for all time [33]. General related results on positivity of wide usefulness are available in $[34,35]$.

The paper is organized as follows. Firstly, a notation and terminology subsection is allocated below in this introductory section. Section 2 characterizes the class of hybrid systems dealt with and formulates with explicit results its positivity and some of its stability and asymptotic stability properties. A relevant auxiliary system for those studies is the so-called extended discrete hybrid system for which only the signals at sampling points are relevant and whose state is composed of both the digital substate and the discretized version of the continuous-time subsystem at sampling instants. Some of the obtained results display how the stability is kept under small coupling between the continuous-time and the discrete-time digital substates provided that the continuoustime and digital dynamics are stable. The section contains also controllability results provided that a nominal system version keeps that property. Section 3 is devoted to the continuous and discrete versions of the KYP-Lemma for a simplified version related to the relevant pairs of the system and control matrices and for the general version related to the whole state-space realization. The relationships between the positive realness of the transfer matrix to the state-space realization are characterized for both the positive extended discrete hybrid system and the whole hybrid system through the KYP-Lemma and Youla's factorization lemma. The obtained results are formulated in terms of robustness in the sense that the positive realness and the system's positivity of a nominal version of the hybrid system are kept under certain explicit conditions for the parametrical disturbances which deviate the hybrid system from its nominal parameterization. Section 4 relates the former results of positive realness and the hyperstability and asymptotic hyperstability properties of the auxiliary extended discrete hybrid system and to those of the whole hybrid system for the case when the plant input is got via feedback from a nonlinear and eventually time-varying device which satisfies a Popov's type inequality. Some further study is also provided in Section 5 related to the design of a stabilizing linear control scheme which either simply stabilizes the dynamics or improves its relative stability degree of the hybrid system in an internal control loop prior to the operation via any member of the given class of nonlinear and time-varying control controllers so as to ensure the hyperstability of the whole closed-loop system. Finally, conclusions end the paper.

\subsection{Notation and Terminology. (a) $\mathbf{R}_{+}$is the set of nonnegative} real numbers; $\mathbf{R}_{+}^{p}$ ( $p$ being a positive integer) is the Cartesian product $p$ times of $\mathbf{R}_{+}$. The vector function $v(t) \in \mathbf{R}_{+}^{p}$ for some $t \geq 0$ if all its components are nonnegative at $t$. The matrix $Q \in \mathbf{R}_{+}^{m \times n}$ if it is of order $m \times n$, with all its entries being nonnegative. $\mathbf{R}_{-}=\mathbf{R} / \mathbf{R}_{+}$is the set of nonpositive real numbers. Note that $\mathbf{R}=\mathbf{R}_{+} \cup \mathbf{R}_{-}$and $0 \in\left(\mathbf{R}_{+} \cap \mathbf{R}_{-}\right)$. Vectors and matrices are nonpositive (being, respectively, in $\mathbf{R}_{-}^{p}$ and $Q \in \mathbf{R}_{-}^{m \times n}$ ) if they have nonpositive entries. $\mathbf{Z}, \mathbf{Z}_{+}$, and $\mathbf{Z}_{-}$are the set of integer numbers and its subsets of nonnegative and nonpositive real parts, respectively.

(b) A matrix $Q \in \mathbf{R}_{+}^{m \times n}$ is said to be positive (denoted by $Q>0$ ) if it has at least a positive entry. A nonnegative matrix $Q \geq 0$ satisfies either $Q>0$ or $Q=0$. A matrix $Q \in \mathbf{R}_{-}^{m \times n}$, which has at least a negative entry, is said to be negative and denoted by $Q<0$ and, if all its entries are negative, then it is denoted by $Q \ll 0$.

(c) A matrix $Q \in \mathbf{R}_{+}^{m \times n}$ is said to be strictly positive (denoted by $Q \gg 0$ ) if all its entries are positive. Similarly, a vector $v \in \mathbf{R}_{+}^{p}$ is said to be positive (denoted by $v>0$ ) if it has at least a positive component. It is said to be strictly positive (denoted by $v \gg 0$ ) if all its components are positive. Also, the notations $A \gg B, v \gg w$ for matrices and vectors mean, respectively, $A-B \gg 0$ and $v-w \gg 0$. Interpretations of expressions like $A>B, v>w, v \geq w$ follow directly from the above ones.

(d) We denote $Q \succ 0(Q \geq 0)$ if $Q \in \mathbf{R}^{n \times n}$ is positive definite (positive semidefinite) and $Q \prec 0(Q \preceq 0)$ if $Q \in \mathbf{R}^{n \times n}$ is negative definite (negative semidefinite).

(e) $I_{n}$ is the $n$th identity matrix.

(f) A matrix $Q \in \mathbf{R}_{+}^{n \times n}$ is said to be stable, or a stability matrix, if its characteristic polynomial is Hurwitz or, equivalently, if all its eigenvalues have negative real parts. The matrix measure of the matrix $Q$ (with respect to any norm $)$ is $\mu(Q)=\lim _{\varepsilon \rightarrow 0^{+}}((\|I+\varepsilon Q\|-\|I\|) / \varepsilon)$. The spectrum of $Q$ is the set of its eigenvalues (or spectrum) denoted by $\mathrm{SpQ}$ and its characteristic polynomial denoted by $p_{\mathrm{Q}}(s)=$ $\operatorname{Det}(s I-Q)$, where $s$ is a complex indeterminate and $\operatorname{Det}(\cdot)$ stands for the determinant of the matrix (.). A subscript in the matrix measure $\mu_{(\cdot)}(Q)$ denotes the measure with respect to a particular (.)-norm. A matrix $Q \in \mathbf{R}_{+}^{n \times n}$ is said to be convergent (or Schur), if all its eigenvalues lie in the strict unity circle. An $H_{\infty}$ complex function is Schur if its $H_{\infty}$ norm is bounded by unity while it is said to be strictly bounded real (SBR), if in addition its coefficients are real and its $H_{\infty}$-norm is strictly bounded by unity.

(g) $K_{p}(Q)=\left\|P_{p}\right\|\left\|P_{p}^{-1}\right\| \in[1, \infty]$ is the condition number of the matrix $Q \in \mathbf{R}^{n \times n}$ with respect to the $p$-norm. It is infinity if and only if the matrix $P^{T} P$ is singular. In particular, $K_{2}(Q)=\left\|Q_{2}\right\|\left\|Q^{-1}\right\|_{2}$ is the condition number of $Q$ with respect to its $\ell_{2}$ (or spectral) norm which is the quotient of its maximum and minimum eigenvalues in the case when it is square.

(h) A matrix $Q=\left(Q_{i j}\right) \in \mathbf{R}^{n \times n}$ is said to be a $n$-Metzler matrix, denoted by $Q \in M_{E}^{n \times n}$, if and only if all its off-diagonal entries satisfy $Q_{i j} \geq 0$ for all $i, j(\neq i) \in \bar{n}:=\{1,2, \ldots, n\}$. A matrix $Q=\left(Q_{i j}\right) \in \mathbf{R}^{n \times n}$ is said to be a $M$-matrix of order $n$, denoted by $Q \in M_{M}^{n \times n}$, if and only if it is a $Z$-matrix; that is, all its entries satisfy $Q_{i i} \geq 0$ and $Q_{i j} \leq 0$ for all $i, j(\neq i) \in \bar{n}=$ $\{1,2, \ldots, n\}$ and, furthermore, all its eigenvalues are strictly unstable.

(i) $Q_{i}$ and $Q_{i}^{T}$ denote, respectively, the $i$ th column or row of the real $Q$-matrix, the superscripts " $T$ " and "*" denoting transpose and conjugate transpose, respectively. $Q^{i}, i$ being an integer number, denotes the $i$ th power of the $Q$-matrix and 
provided that $Q=\left(Q_{i j}\right), Q^{(G)}=\left(Q_{i j}^{(G)}\right)$ is an associate matrix to $Q$ defined as $Q_{i j}^{(G)}=1$ if $Q_{i j} \neq 0$ and $Q_{i j}^{(G)}=0$, otherwise. Note that $Q \geq 0 \Leftrightarrow Q^{(G)} \geq 0 . v_{i}$ denotes the $i$ th component of the real vector $v$ and $v \geq 0 \Leftrightarrow v^{(G)} \geq 0$. Thus, any positive system $S$ has always an associate positive system $S^{(G)}$ which defines the pairwise relations input components/state-output components and state components/output components from its associate influence graph $G[2,3,5]$, by defining all its parameterizing matrices according to the above criterion.

(j) $U(t)=1(t)$ is the unity step (Heaviside) function.

(k) $e_{j}^{(n)}$ is the unity vector of $\mathbf{R}^{n}$ whose unique nonzero component is the $j$ th one which is unity.

(l) The notation $x[k]$ stands for a discrete/digital variable or vector $x$ which is only defined as sampling instants $t_{k}=$ $k T, k \in \mathbf{Z}_{+}$, with $T$ being the sampling period. If $x$ is a digital variable then it is only defined at sampling instants. If $x$ is a discrete variable (i.e., that arising from the discretization of a continuous variable), then $x[k]=x(k T)$ and any of both equivalent notations are used indistinctly in such a case.

(m) The superscript $T$ stands for the transpose of a vector or matrix while $\operatorname{Ker}(O)$ stands for the null-space of the operator $O$.

\section{Hybrid System and Positivity and Controllability Properties}

Consider the subsequent hybrid linear system $H$ :

$$
\begin{aligned}
\dot{x}_{c}(t)= & A_{c} x_{c}(t)+A_{c s} x_{c}[k]+A_{c d} x_{d}[k] \\
& +B_{c} u(t)+B_{c s} u[k], \\
x_{d}[k+1]= & A_{d} x_{d}[k]+A_{d s} x_{c}[k]+B_{d} u[k], \\
y(t)= & C_{c} x_{c}(t)+C_{c s} x_{c}[k]+C_{d} x_{d}[k]+D_{c} u(t) \\
& +D_{d} u[k]
\end{aligned}
$$

for all $t \in[k T,(k+1) T)$ for any integer $k \geq 0$ with $T>0$ being the sampling period, where $x_{c}$ and $x_{d}$ are, respectively, the continuous and digital substates of respective dimensions $n_{c}$ and $n_{d}$, and $u \in \mathbf{R}^{m}$ and $y \in \mathbf{R}^{p}$ are the input and output vectors. The continuous-time argument is denoted by $(t)$ while the discrete-time argument is denoted by $[k]$ and the associated continuous and digital variables are denoted correspondingly. That is, a continuous variable at sampling instants is denoted in the same way as a purely digital variable such that continuous and discrete (or digital) time arguments are, respectively, denoted with parenthesis $(\cdot)$, such as $x_{c}(t), y(t)$, and $u(t)$, and brackets [.], such as $x_{c}[k]=x_{c}(k T), x_{d}[k], u[k]=u(k T)$. On the other hand, the parameterization of (1a), (1b), and (1c) is as follows:

(i) $A_{c}$ and $A_{d}$ are the matrix of continuous-time and of digital dynamics, respectively, and $A_{c d}$ and $A_{d s}$ are, respectively, the matrices of dynamics of couplings between the digital and continuous-time substates and continuoustime discretized and digital substates. The matrix $A_{c s}$ is the matrix of dynamics of coupling between the sampled continuous-time substate to its time evolution over the next sampling interval. (ii) $B_{c}$ and $B_{d}$ are continuous-time and digital control matrices and $B_{c s}$ is a coupling control matrix from the sampled continuous-time control to the next intersample period continuous-time substate.

(iii) The matrices $C_{c}, C_{c s}, C_{d}$ and $D_{c}$ and $D_{d}$ in (1c) are the various output and input-output interconnection matrices generating the output of the hybrid system from its continuous-time substate, its discretized value at sampling instants, the digital substate, and the continuous-time input and its sampled value.

The orders of all the real constant system parameterizing matrices displayed in (1a), (1b), and (1c) agree with the corresponding dimensions of the continuous, discrete, and digital substates $x_{c}(t), x_{c}[k]$, and $x_{d}[k]$ and inputs and outputs. Note that the hybrid system is driven by the control $u(t)$ and by its samples $u(k T)$ of period $T$ acting as two independent control actions. At sampling instants, it follows by direct calculus from (1a), (lb), and (1c) that the hybrid system $H$ is described by the following $n=n_{c}+n_{d}$ th order extended discrete-time system of sampling period $T$ driven by a fictitious extended input sequence $\{v[k]\} \subset \mathbf{R}^{m+n}$ whose element $v[k]$ depends on $u:[k T,(k+1) T] \rightarrow \mathbf{R}^{m}$ and since only finite input jumps happen at sampling instants, since impulsive jumps are not considered, $v[k]$ depends on $u:[k T,(k+1) T) \rightarrow \mathbf{R}^{m}$ since the updated value $u[k+1]$ at $t=(k+1) T$ does not contribute to $v[k] D H$ :

$$
\begin{aligned}
x[k+1] & =A x[k]+B_{0} u[k]+\xi[k], \\
& =A x[k]+B_{v} v[k], \\
y[k] & =C x[k]+D u[k]
\end{aligned}
$$

of state $x[k]=\left[x_{c}^{T}[k], x_{d}^{T}[k]\right]^{T}$ for any integer $k \geq 0$, where

$$
\begin{aligned}
A & =\left[\begin{array}{ll}
A_{c}^{\prime} & A_{d}^{\prime} \\
A_{d s} & A_{d}
\end{array}\right] ; \\
B_{0} & =\left[\begin{array}{c}
e^{A_{c} T}\left(\int_{0}^{T} e^{-A_{c} \tau} d \tau\right) B_{c s} \\
B_{d}
\end{array}\right], \\
A_{c}^{\prime} & =e^{A_{c} T}\left[I_{n_{c}}+\left(\int_{0}^{T} e^{-A_{c} \tau} d \tau\right) A_{c s}\right] ; \\
A_{d}^{\prime} & =e^{A_{c} T}\left(\int_{0}^{T} e^{-A_{c} \tau} d \tau\right) A_{c d}, \\
C & =\left[\begin{array}{cc}
C_{c}+C_{c s} & \vdots C_{d}
\end{array}\right] ; \\
D & =D_{c}+D_{d}, \\
B_{v} & =\left[B_{0} \vdots \begin{array}{c}
I_{n_{c}} \\
n_{n_{d} \times n_{d}}
\end{array}\right] \\
\xi[k] & =\left[\xi_{c}^{T}[k], 0^{T}\right]^{T} ; \\
\xi_{c}[k] & =e^{A_{c} T}\left(\int_{0}^{T} e^{-A_{c} \tau} B_{c} u(k T+\tau) d \tau\right),
\end{aligned}
$$




$$
v[k]=\left[u^{T}[k], \xi_{c}^{T}[k]\right]^{T},
$$

where $v[k] \in \mathbf{R}^{m+n}$. The derivation of the extended discrete $\mathrm{DH},(2 \mathrm{a}),(2 \mathrm{~b})$, and (2c), subject to (3)-(8), from the hybrid system $H,(1 \mathrm{a}),(1 \mathrm{~b})$, and (1c), is direct from a time-integration of (1a), (1b), and (1c) on a sampling time interval $[k T,(k+$ 1) $T$ ) with initial conditions at $t=k T$. The following positivity result holds as a direct extension from the SISO (single-input single-output case) hybrid parameterization of $[7,11,31]$.

Theorem 1. The system $H$ is positive if and only if $A_{c} \in M_{E}^{n_{c} \times n_{c}}$, $A_{c s} \in \mathbf{R}_{+}^{n_{c} \times n_{c}}, A_{c d} \in \mathbf{R}_{+}^{n_{c} \times n_{d}}, A_{d} \in \mathbf{R}_{+}^{n_{d} \times n_{d}}, A_{d s} \in \mathbf{R}_{+}^{n_{d} \times n_{c}}, B_{c} \in$ $\mathbf{R}_{+}^{n_{c} \times m}, B_{c s} \in \mathbf{R}_{+}^{n_{c} \times m}, B_{d} \in \mathbf{R}_{+}^{n_{d} \times m}, C_{c} \in \mathbf{R}_{+}^{p \times n_{c}}, C_{c s} \in \mathbf{R}_{+}^{p \times n_{c}}$, $C_{d} \in \mathbf{R}_{+}^{p \times n_{d}}, D_{c} \in \mathbf{R}_{+}^{p \times m}, D_{d} \in \mathbf{R}_{+}^{p \times m}$.

Under the above given conditions, $e^{A_{c} T} \in \mathbf{R}_{+}^{n_{c} \times n_{c}}, A_{c}^{\prime} \in$ $\mathbf{R}_{+}^{n_{c} \times n_{c}}, A_{d}^{\prime} \in \mathbf{R}_{+}^{n_{c} \times n_{d}}, B_{0} \in \mathbf{R}_{+}^{n \times 2 m}, C \in \mathbf{R}_{+}^{p \times n}, D \in \mathbf{R}_{+}^{p \times m}$, $\xi[k] \in \mathbf{R}_{+}^{n}$, and $v[k] \in \mathbf{R}_{+}^{m+n}$ for $k \geq 0$, if $u:[0, \infty] \rightarrow \mathbf{R}^{m}$, and then the extended discrete system $\mathrm{DH}$ is also positive.

Theorem 2. The following properties hold:

(i) Assume that

(a) $A_{c} \in M_{E}^{n_{c} \times n_{c}}, A_{c s} \in \mathbf{R}_{+}^{n_{c} \times n_{c}}, A_{c d} \in \mathbf{R}_{+}^{n_{c} \times n_{d}}, A_{d} \in \mathbf{R}_{+}^{n_{d} \times n_{d}}$, $A_{d s} \in \mathbf{R}_{+}^{n_{d} \times n_{c}}, B_{c} \in \mathbf{R}_{+}^{n_{c} \times m}, B_{c s} \in \mathbf{R}_{+}^{n_{c} \times m}, B_{d} \in \mathbf{R}_{+}^{n_{d} \times m}$,

(b) $A_{c}^{\prime}, A_{d}, A_{c}^{\prime}+A_{d}^{\prime}\left(I_{n_{d}}-A_{d}\right)^{-1} A_{d s}$ and $A_{d}+A_{d s}\left(I_{n_{c}}-\right.$ $\left.A_{c}^{\prime}\right)^{-1} A_{d}^{\prime}$ are convergent.

Then, $A$ is convergent and the unforced DH is globally asymptotically stable.

(ii) Under the hypotheses of Property (i), assume also that $A$ is convergent, $A>0$, and that $1 /\left\|\left(I_{n}-A\right)^{-1}\right\|_{2}>\|(A-$ $\left.A_{a d}\right) \|_{2}$, where $A_{a d}=\operatorname{Block} \operatorname{Diag}\left(A_{c}^{\prime}, A_{d}\right)$. Then, $A_{c}^{\prime}$ and $A_{d}$ are convergent.

(iii) $A$ is convergent, and then the unforced $D H$ and the unforced $H$ are both globally asymptotically stable, if the following conditions hold:

(1) $A_{c}$ is Hurwitz and $A_{d}$ is convergent such that $\rho_{d}^{-1} \leq$ $\left\|A_{d}\right\|_{2} \leq \rho_{d 1}$ and $e^{-\rho_{c} t} \leq\left\|e^{A_{c} t}\right\|_{2} \leq e^{-\rho_{c 1} t}$ for some real constants $\rho_{c}, \rho_{c 1}, \rho_{d 1}$, and $\rho_{d}$, with $\rho_{c} \geq \rho_{c 1}>0$ and $\rho_{d}^{-1} \leq$ $\rho_{d 1}<1$.

(2) $\left\|A_{c s}\right\|_{2} \leq \varepsilon,\left\|A_{d s}\right\|_{2} \leq \varepsilon$, and $\left\|A_{c d}\right\|_{2} \leq \varepsilon$ for some $\varepsilon \in\left[0, \varepsilon^{*}\right)$, where $\varepsilon^{*}=\rho_{c} / 2 \sqrt{3}\left[\left(1-e^{\rho_{c} T}\right)^{2}+\rho_{d}^{2} \rho_{c}^{2}\right]^{1 / 2}$.

(iv) $A$ is convergent if $A_{c}^{\prime}$ and $A_{d}$ are convergent and $\left\|A_{c d}\right\|_{2}<1 /\left\|A_{c}^{\prime-1}\left(\int_{0}^{T} e^{A_{c}(T-\tau)} d \tau\right)\right\|_{2}\left(1+\left\|A_{d}^{-1} A_{d s}\right\|_{2}\right)$.

Proof. First note that $A>0$ and is convergent if and only if $(I-A)$ is nonsingular and $(I-A)^{-1}>0$ [9]. Direct calculations with (3) and the inverse of a $2 \times 2$ block partitioned matrix [36] yield in this case

$$
\begin{aligned}
I-A & =\left[\begin{array}{cc}
I_{n}-A_{c}^{\prime} & -A_{d}^{\prime} \\
-A_{d s} & I_{n}-A_{d}
\end{array}\right], \\
(I-A)^{-1} & =\left[\begin{array}{ll}
\Gamma_{11} & \Gamma_{12} \\
\Gamma_{21} & \Gamma_{22}
\end{array}\right],
\end{aligned}
$$

where

$$
\Gamma_{11}=\left[I_{n_{c}}-A_{c}^{\prime}-A_{d}^{\prime}\left(I_{n_{d}}-A_{d}\right)^{-1} A_{d s}\right]^{-1},
$$

$$
\begin{aligned}
\Gamma_{12} & =\left(I_{n_{c}}-A_{c}^{\prime}\right)^{-1} \\
\cdot & A_{d}^{\prime}\left[I_{n_{d}}-A_{d}-A_{d s}\left(I_{n_{c}}-A_{c}^{\prime}\right)^{-1} A_{d}^{\prime}\right]^{-1} \\
& =\left[I_{n_{c}}-A_{c}^{\prime}-A_{d}^{\prime}\left(I_{n_{d}}-A_{d}\right)^{-1} A_{d s}\right]^{-1} \\
\cdot & A_{d}^{\prime}\left(I_{n_{d}}-A_{d}\right)^{-1}, \\
\Gamma_{21} & =\left(I_{n_{d}}-A_{d}\right)^{-1} \\
\cdot & A_{d s}\left[I_{n_{c}}-A_{c}^{\prime}-A_{d}^{\prime}\left(I_{n_{d}}-A_{d}\right)^{-1} A_{d s}\right]^{-1} \\
& =\left[I_{n_{d}}-A_{d}-A_{d s}\left(I_{n_{c}}-A_{c}^{\prime}\right)^{-1} A_{d}^{\prime}\right]^{-1} \\
\cdot & A_{d s}\left(I_{n_{c}}-A_{c}^{\prime}\right)^{-1}, \\
\Gamma_{22}= & {\left[I_{n_{d}}-A_{d}-A_{d s}\left(I_{n_{d}}-A_{c}^{\prime}\right)^{-1} A_{d}^{\prime}\right]^{-1} \cdot }
\end{aligned}
$$

Note that the above matrices exist with $\Gamma_{i j}>0, i, j=1,2$, since all the needed intermediate matrix inverses exist since

(a) $A_{d}$ and $A_{c}^{\prime}$ are convergent, $A_{d}>0$, and $A_{c}^{\prime}>0$ (since $A_{c} \in M_{E}^{n_{c} \times n_{c}}$, and then $e^{A_{c} t}>0$ for any real $\left.t \geq 0\right)$ and $A_{c s} \epsilon$ $\mathbf{R}_{+}^{n_{c} \times n_{c}}$, so that $\left(I_{n_{d}}-A_{d}\right)$ and $\left(I_{n_{c}}-A_{c}^{\prime}\right)$ are nonsingular with $\left(I_{n_{d}}-A_{d}\right)^{-1}>0$ and $\left(I_{n_{c}}-A_{c}^{\prime}\right)>0$,

(b) $A_{c}^{\prime}+A_{d}^{\prime}\left(I_{n_{d}}-A_{d}\right)^{-1} A_{d s}$ and $A_{d}+A_{d s}\left(I_{n_{c}}-A_{c}^{\prime}\right)^{-1} A_{d}^{\prime}$ are convergent with nonnegative entries so that $\Gamma_{11}$ exists, $\Gamma_{11}>0$, and $\left[I_{n_{d}}-A_{d}-A_{d s}\left(I_{n_{c}}-A_{c}^{\prime}\right)^{-1} A_{d}^{\prime}\right]^{-1}$ with $\left[I_{n_{d}}-A_{d}-A_{d s}\left(I_{n_{c}}-\right.\right.$ $\left.\left.A_{c}^{\prime}\right)^{-1} A_{d}^{\prime}\right]^{-1}>0$.

As a result, there exists $(I-A)^{-1}$ with $A>0$ and $(I-$ $A)^{-1} \geq 0$ which holds if and only if $A$ is convergent so that the unforced $D H$ is globally asymptotically stable. Property (i) has been proved. To prove Property (ii), note that $A>0$ and is convergent, so that $\left(I_{n}-A\right)$ is a nonsingular $M$-matrix and $\left(I_{n}-A\right)^{-1}>0$ exists. Since $\left(I_{n}-A\right)$ is a nonsingular $M$ matrix, all its leading minors are positive. Thus, $\left(I_{n}-A_{a d}\right)>0$, then $\left(I_{n_{c}}-A_{c}^{\prime}\right)>0$ and $\left(I_{n_{d}}-A_{d}\right)>0$ and, equivalently, $\left(I_{n_{c}}-A_{c}^{\prime}\right)^{-1}>0$ and $\left(I_{n_{d}}-A_{d}\right)^{-1}>0$. On the other hand, one has

$$
\begin{aligned}
& \left(I_{n}-A_{a d}\right)^{-1} \\
& \quad=\left[I_{n}-\left(I_{n}-A\right)^{-1}\left(A-A_{a d}\right)\right]^{-1}\left(I_{n}-A\right)^{-1} .
\end{aligned}
$$

Since $A \geq A_{a d}$ from the hypotheses, $\left(I_{n}-A\right)^{-1}\left(A-A_{a d}\right) \geq 0$ and if $\left(I_{n}-A\right)^{-1}\left(A-A_{a d}\right)$ is convergent, that is, if $1>\|\left(I_{n}-\right.$ $A)^{-1}\left(A-A_{a d}\right) \|_{2}$ guaranteed if $1 /\left\|\left(I_{n}-A\right)^{-1}\right\|_{2}>\left\|\left(A-A_{a d}\right)\right\|_{2}$, then $\left[I_{n}-\left(I_{n}-A\right)^{-1}\left(A-A_{a d}\right)\right]^{-1}>0$. As a result, $\left(I_{n}-A_{a d}\right)^{-1}>$ $0,\left(I_{n_{c}}-A_{c}^{\prime}\right)>0$, and $\left(I_{n_{d}}-A_{d}\right)>0$ and they have real positive eigenvalues since $\left(I_{n_{c}}-A_{c}^{\prime}\right)>0$ and $\left(I_{n_{d}}-A_{d}\right)>0$. Thus, for any given nonzero vectors $z_{c}$ and $z_{d}$ of respective dimensions $n_{c}$ and $n_{d}$, one concludes that $z_{c}^{T} z_{c}>z_{c}^{T} A_{c}^{\prime} z_{c}$ and $z_{d}^{T} z_{d}>$ $z_{c}^{T} A_{d} z_{d}$. This implies that the spectral radii of $A_{c}^{\prime}$ and $A_{d}$ are less than unity so that $A_{c}^{\prime}$ and $A_{d}$ are positive and convergent. 
To prove Property (iii), note that since $A_{c}$ is Hurwitz and $A_{d}$ is convergent, $A=A_{0}+\widetilde{A}=A_{0}\left(I_{2 n}+A_{0}^{-1} \widetilde{A}\right)$, where

$$
\begin{aligned}
& A_{0}=\text { Block Diag }\left(e^{A_{c} T}: A_{d}\right) \\
& \widetilde{A}=\left[\left(\int_{0}^{T} e^{A_{c}(T-\tau)} d \tau\right) A_{c s}\left(\int_{0}^{T} e^{A_{c}(T-\tau)} d \tau\right) A_{c d}\right]
\end{aligned}
$$

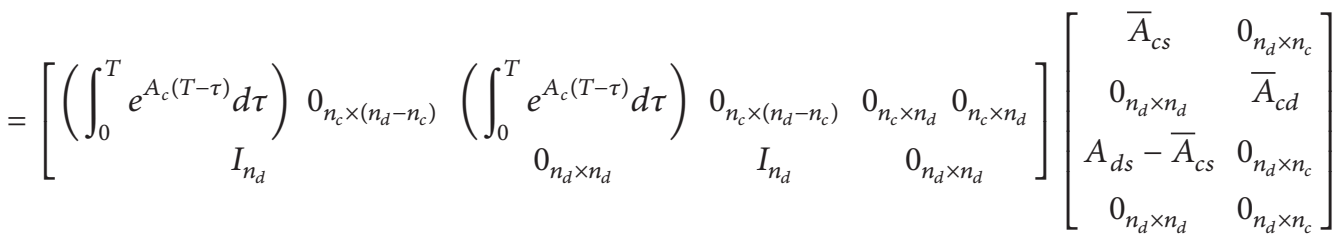

with

$$
\begin{aligned}
& \bar{A}_{c s}=\left[\begin{array}{c}
A_{c s} \\
0_{\left|n_{d}-n_{c}\right| \times n_{c}}
\end{array}\right] \in \mathbf{R}^{n_{d} \times n_{c}} ; \\
& \bar{A}_{c d}=\left[\begin{array}{c}
A_{c d} \\
0_{\left|n_{d}-n_{c}\right| \times n_{c}}
\end{array}\right] \in \mathbf{R}^{n_{d} \times n_{c}}, \\
& A_{0}^{-1} \widetilde{A}=\left(\begin{array}{cc}
e^{-A_{c} T} & 0_{n_{c} \times n_{d}} \\
0_{n_{d} \times n_{c}} & A_{d}^{-1}
\end{array}\right)[L, L, 0]\left[\begin{array}{cc}
\bar{A}_{c s} & 0_{n_{d} \times n_{c}} \\
0_{n_{d} \times n_{d}} & \bar{A}_{c d} \\
A_{d s}-\bar{A}_{c s} & 0_{n_{d} \times n_{c}} \\
0_{n_{d} \times n_{d}} & 0_{n_{d} \times n_{c}}
\end{array}\right]
\end{aligned}
$$

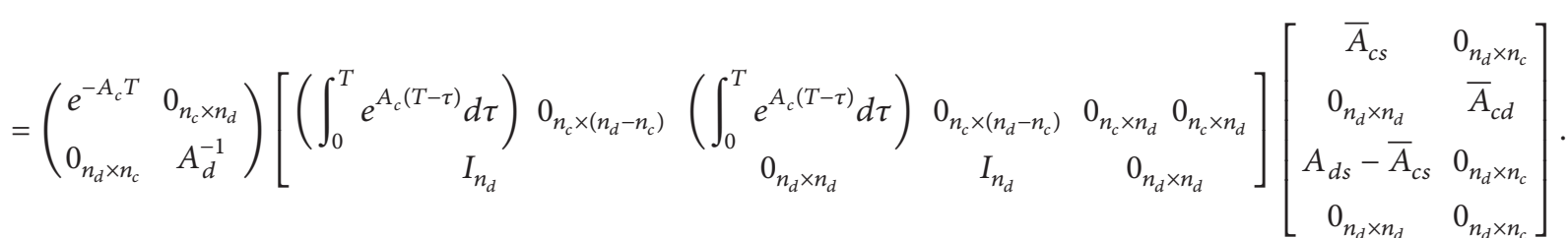

Since $A_{c}$ is Hurwitz and $A_{d}$ and $A_{0}$ are convergent, there exists a real constant $K \geq 1$, which is norm-dependent such that $K e^{-\rho_{c} t} I_{n} \leq e^{A_{c} t} \leq K e^{-\rho_{c 1} t} I_{n}$ for all $t \geq 0$. Note that $1 \leq\left\|A_{d}^{-1}\right\|_{2} \leq \rho_{d}$ and $e^{\rho_{c 1} t} \leq\left\|e^{-A_{c} t}\right\|_{2} \leq e^{\rho_{c} t}$ for all $t \geq 0$ with $-\rho_{c 1}<0$ being not less than the stability abscissa of $A_{c}$ and $\rho_{d 1}$ being not less than the convergence abscissa of $A_{d}$. Since $\max \left(\left\|A_{c s}\right\|_{2},\left\|A_{d s}\right\|_{2},\left\|A_{c d}\right\|_{2}\right) \leq \varepsilon$, one gets from (15) that

$$
\begin{aligned}
\left\|A_{0}^{-1} \widetilde{A}\right\|_{2} & =\left\|\left(A_{0}^{-1} \widetilde{A}\right)^{T}\left(A_{0}^{-1} \widetilde{A}\right)\right\|_{2}^{1 / 2} \\
& \leq\left(2\left\|L^{T} L\right\|_{2}^{1 / 2}\right)\left(\sqrt{3 \varepsilon^{2}}\right) \\
& \leq 2 \sqrt{3}\left(\frac{\left(1-e^{\rho_{c} T}\right)^{2}}{\rho_{c}^{2}}+\rho_{d}^{2}\right)^{1 / 2} \varepsilon .
\end{aligned}
$$

If $\varepsilon \in\left[0, \varepsilon^{*}\right)$, then $A=A_{0}\left(I_{2 n}+A_{0}^{-1} \widetilde{A}\right)$ is nonsingular from Banach Perturbation Lemma [37] and then convergent since $A_{0}$ is convergent from the continuity of the eigenvalues of matrix with respect to its entries. Then the unforced $D H$ is globally asymptotically stable and the unforced $H$ is also globally asymptotically stable since $A_{c}$ is Hurwitz and $x[k] \rightarrow$ 0 as $k \rightarrow \infty$ for any given initial condition. Property (iii) has been proved. Property (iv) follows by redefining $A=$ $A_{01}+\widetilde{A}_{1}=A_{01}\left(I_{2 n}+A_{01}^{-1} \widetilde{A}_{1}\right)$

$$
\begin{aligned}
A_{01} & =\left[\begin{array}{cc}
A_{c}^{\prime} & 0_{n_{c} \times n_{d}} \\
A_{d s} & A_{d}
\end{array}\right] ; \\
A_{01}^{-1} & =\left[\begin{array}{cc}
A_{c}^{\prime-1} & 0_{n_{c} \times n_{d}} \\
-A_{d}^{-1} A_{d s} A_{c}^{\prime-1} & A_{d}^{-1}
\end{array}\right] ;
\end{aligned}
$$




$$
\widetilde{A}_{1}=\left[\begin{array}{cc}
0_{n_{d} \times n_{c}} \\
0_{n_{d} \times n_{c}}
\end{array}\left(\int_{0}^{T} e^{A_{c}(T-\tau)} d \tau\right) A_{c d}\right]
$$

and then

$$
\begin{aligned}
& A_{01}^{-1} \widetilde{A}_{1} \\
& =\left[\begin{array}{cc}
A_{c}^{\prime-1} & 0_{n_{c} \times n_{d}} \\
-A_{d}^{-1} A_{d s} A_{c}^{\prime-1} & A_{d}^{-1}
\end{array}\right]\left[\begin{array}{c}
0_{n_{d} \times n_{c}}\left(\int_{0}^{T} e^{A_{c}(T-\tau)} d \tau\right) A_{c d} \\
0_{n_{d} \times n_{c}} \\
0_{n_{d} \times n_{d}}
\end{array}\right] \\
& =\left[\begin{array}{cc}
0_{n_{c} \times n_{c}} & A_{c}^{\prime-1}\left(\int_{0}^{T} e^{A_{c}(T-\tau)} d \tau\right) A_{c d} \\
0_{n_{d} \times n_{c}} & -A_{d}^{-1} A_{d s} A_{c}^{\prime-1}\left(\int_{0}^{T} e^{A_{c}(T-\tau)} d \tau\right) A_{c d}
\end{array}\right]
\end{aligned}
$$

so that $A$ is nonsingular if $\left\|A_{c d}\right\|_{2}<1 /\left\|A_{c}^{\prime^{-1}}\left(\int_{0}^{T} e^{A_{c}(T-\tau)} d \tau\right)\right\|_{2}(1+$ $\left.\left\|A_{d}^{-1} A_{d s}\right\|_{2}\right)$.

Related to Theorem 2, note that $A_{c}^{\prime}$ is convergent by construction if $A_{c}$ is Hurwitz and a guaranteed upper-bound of $\left\|A_{c s}\right\|$ is sufficiently small which increases as the sampling period $T$ and the stability abscissa of $A_{c}$ increase. The next result generalizes Theorem 1 if $A_{d}$ is not necessarily convergent and $A_{c}$ is not necessarily Hurwitz.

Example 3. Consider a positive hybrid system with scalar continuous-time and digital subsystems and $A_{c}=-a_{c}$ such that $A_{c}^{\prime}=a_{c}^{\prime}=e^{-a_{c} T}+\left(1-e^{-a_{c} T}\right) a_{c s} / a_{c}$ and $A_{d}^{\prime}=a_{d}^{\prime}=$ $e^{-a_{c} T}+\left(1-e^{-a_{c} T}\right) a_{c s} / a_{c}, A_{d s}=a_{d s} \geq 0, A_{c s}=a_{c s} \geq 0$, and $A_{d}=a_{d} \in[0,1)$, that is, nonnegative and convergent. From Theorem 2(i) if $A_{c}^{\prime}$ is convergent then $A_{c}$ is convergent implying that $a_{c}>0$. Furthermore, for some $\varepsilon \in(0,1), A_{c}^{\prime}$ is nonnegative and convergent if $0 \leq e^{-a_{c} T}+\left(1-e^{-a_{c} T}\right) a_{c s} / a_{c} \leq$ $1-\varepsilon$ which implies that $a_{c s} \leq\left(1-\varepsilon-e^{-a_{c} T}\right) a_{c} /\left(1-e^{-a_{c} T}\right)$. Note that $a_{c s}<a_{c}$.

On the other hand, hypothesis $a_{c}^{\prime}+a_{d}^{\prime}\left(I_{n_{d}}-a_{d}\right)^{-1} a_{d s}$ being nonnegative and convergent holds if

$$
\begin{aligned}
0 & \leq a_{c}^{\prime}+a_{d}^{\prime}\left(I_{n_{d}}-a_{d}\right)^{-1} a_{d s} \leq 1-\varepsilon_{1} \\
& \leq 1-\varepsilon+a_{d}^{\prime}\left(I_{n_{d}}-a_{d}\right)^{-1} a_{d s}
\end{aligned}
$$

for some $\varepsilon_{1} \in\left(\max \left(\varepsilon-a_{d}^{\prime}\left(I_{n_{d}}-a_{d}\right)^{-1} a_{d s}, 0\right), 1-a_{c}^{\prime}\right)$; that is, the left-hand side is nonnegative convergent, since

$$
0 \leq a_{d s} \leq \frac{a_{c}\left(1-a_{d}\right)\left(1-a_{c}^{\prime}-\varepsilon_{1}\right)}{a_{c d}\left(1-e^{-a_{c} T}\right)}
$$

while $a_{d}+a_{d s}\left(I_{n_{c}}-a_{c}^{\prime}\right)^{-1} a_{d}^{\prime}$ being convergent holds if

$$
\begin{aligned}
0 & \leq a_{d} \leq 1-\varepsilon_{2}-\frac{a_{c} a_{d s} a_{c d}}{\left(1-a_{c}^{\prime}\right) a_{c}} \\
& =1-\varepsilon_{2}-\frac{a_{d s} a_{c d}}{\left(1-e^{-a_{c} T}\right)\left(1-a_{c s} / a_{c}\right)-\varepsilon_{2}}
\end{aligned}
$$

for some $\varepsilon_{2} \in\left(0,\left(1-e^{-a_{c} T}\right)\left(1-a_{c s} / a_{c}\right)\right)$. Particular numerical values which satisfy all the given joint constraints are, for instance, $a_{c}=0.1$, so that the stable continuous dynamics has a small relative stability, $T=0.1, a_{d}=0$, so that the digital dynamics has a maximum stability degree, and the forced system behavior is independent of the digital self-dynamics, $a_{d}^{\prime}=0.995, a_{d s}=0.009$.

It can be verified that $A$ is convergent with eigenvalues 0.9919 and -0.0089 . The unforced $D H$ is globally asymptotically stable [Theorem 2(i)]. Since, in this case with $n_{c}=1$ and $a_{c s} \geq 0, a_{c}^{\prime}$ being convergent implies that $a_{c}>0$ (i.e., $A_{c}=-a_{c}$ is Hurwitz), the unforced $H$ is also globally asymptotically stable [Theorem 2(iii)].

\section{Corollary 4. The following properties hold:}

(i) $A$ is convergent, and then the unforced $D H$ is globally asymptotically stable, if

(1) $A_{0}$, (12), is nonsingular and there exists $\lambda(\in \mathbf{R}) \in$ $(-1,-1 / 2) \cup[0, \infty)$ such that $(1+\lambda) A_{0}$ is convergent,

(2) there exists $\mathbf{R} \ni \lambda_{1} \in\left(|\lambda|,(1+\lambda) / K_{2}\left(A_{0}\right)\right)$ such that $K_{2}\left(A_{0}\right)<(1+\lambda) / \lambda_{1}$ and $\|\widetilde{A}\|_{2}<\left(\lambda_{1}-|\lambda|\right)\left\|A_{0}\right\|_{2}$.

If, in addition, $A_{c}$ is Hurwitz, then the unforced $D H$ is globally asymptotically stable.

(ii) A sufficient condition for Property (i) to hold is

$$
\begin{aligned}
& \left\|\left[\begin{array}{cc}
A_{c s} & A_{c d} \\
A_{d s} & 0_{n_{d} \times n_{d}}
\end{array}\right]\right\|_{2} \\
& \quad<\left(1+\left|\frac{\rho_{c}}{1-e^{\rho_{c} T}}\right|\right)\left(\lambda_{1}-|\lambda|\right)\left\|A_{0}\right\|_{2},
\end{aligned}
$$

where $\rho_{c} \in \mathbf{R}$ is the stability abscissa of $A_{c}$.

Proof. Note that, since $A_{0}$ is nonsingular,

$$
\begin{aligned}
A & =(1+\lambda) A_{0}+\left(\widetilde{A}-\lambda A_{0}\right) \\
& =(1+\lambda) A_{0}\left(I_{2 n}+\frac{1}{1+\lambda} A_{0}^{-1}\left(\widetilde{A}-\lambda A_{0}\right)\right)
\end{aligned}
$$

and $A$ is nonsingular from Banach's Perturbation Lemma, under the condition $\|\widetilde{A}\|_{2}<\left(\lambda_{1}-|\lambda|\right)\left\|A_{0}\right\|_{2}$, if $|\lambda|<\lambda_{1}$ and

$$
\begin{aligned}
1 & >\frac{\lambda_{1}}{1+\lambda} K_{2}\left(A_{0}\right)=\frac{\lambda_{1}}{1+\lambda}\left\|A_{0}\right\|_{2}\left\|A_{0}^{-1}\right\|_{2} \\
& >\frac{1}{1+\lambda}\left\|A_{0}^{-1}\right\|_{2}\left(\|\widetilde{A}\|_{2}+|\lambda|\left\|A_{0}\right\|_{2}\right) \\
& >\frac{1}{1+\lambda}\left\|A_{0}^{-1}\right\|_{2}\left\|\widetilde{A}-\lambda A_{0}\right\|_{2} .
\end{aligned}
$$

Since $|\lambda|<\lambda_{1}$ and $1 \leq K_{2}\left(A_{0}\right)<(1+\lambda) / \lambda_{1}$, then $|\lambda|<\lambda_{1}<$ $1+\lambda$ and, if $\lambda<0$, then $|\lambda|<1 / 2$ and $|\lambda|<\lambda_{1}<1-|\lambda|$. Property (i) follows directly from (24). Property (ii) follows from the fact that (22) is a sufficient condition for $\|\widetilde{A}\|_{2}<$ $\left(\lambda_{1}-|\lambda|\right)\left\|A_{0}\right\|_{2}$ in view of the first identity of (13).

The following theorem refers to "controllability" as the property of controllability to the origin and to "reachability" as that of controllability from the origin. Note from (2a), (3), 
and (6)-(8) the structure of matrix $B$ that $B_{0} u[k]+\xi[k]=$ $B_{v} v[k]=B g[k]$ leading to the state system $D H$ description driven by a real vector sequence:

$$
\begin{aligned}
x[k+1] & =A x[k]+B_{v} v[k] \\
& =A_{g} x[k]+B_{g} v[k]+\delta_{v}[k] \\
& =A_{g} x[k]+B_{g} g[k]+\delta[k] \\
y[k] & =C x[k]+D g[k] \\
& =C_{g} x[k]+D_{g} g[k]+\delta_{y}[k]
\end{aligned}
$$

for any integer $k \geq 0$, where $B_{v}$ is reparameterized to some appropriate matrix $B$ so as to drive the auxiliary control $\{g[k]\}_{k \in \mathbf{Z}_{0+}} \subset \mathbf{R}^{m+n}$ of the form $g[k]=\left(u^{T}[k], g_{c}^{T}[k]\right)^{T}$, where $\left\{g_{c}[k]\right\}_{k \in \mathbf{Z}_{0+}} \subset \mathbf{R}^{n}$, as follows:

$$
\begin{aligned}
& u(k T+\tau)=V B_{c}^{T} e^{A_{c}^{T}(T-\tau)} g_{c}[k], \\
& \forall \tau \in(0, T) ; u(k T)=u[k]
\end{aligned}
$$

for some given prefixed $m \times m$-matrix $V=V^{T} \geq 0$, and

$$
\begin{aligned}
& \delta_{v}[k]=\left(A-A_{g}\right) x[k]+\left(B-B_{g}\right) v[k] \\
& \delta[k]=\left(A-A_{g}\right) x[k]+\left(B-B_{g}\right) g[k], \\
& \delta_{y}[k]=\left(C-C_{g}\right) x[k]+\left(D-D_{g}\right) g[k], \\
& B_{g} \\
& =\left[\left(\int_{0}^{T} e^{A_{g c}(T-\tau)} d \tau\right) B_{g c s} \int_{0}^{T} e^{A_{g c}(T-\tau)} B_{g c} V B_{g c}^{T} e^{A_{g c}^{T}(T-\tau)} d \tau\right] \\
& B=\left[\left(\int_{0}^{T} e^{A_{c}(T-\tau)} d \tau\right) B_{c s} \int_{0}^{T} e^{A_{c}(T-\tau)} B_{c} V B_{c}^{T} e^{A_{c}^{T}(T-\tau)} d \tau\right] \\
& D_{g}=D_{g c}+D_{g d} ; \\
& D=D_{c}+D_{d} .
\end{aligned}
$$

Theorem 5. Define $\widetilde{A}_{g}=A-A_{g}$ and $\widetilde{B}_{g}=B-B_{g}$ such that $\left(A_{g}, B_{g}\right)$ is a nominal controllable pair and $B_{g}=\{X \in$ $\left.\mathbf{R}_{+}^{n \times\left(n_{c}+m\right)}:\left\|\widetilde{B}_{g}\right\|_{2} \leq Y, \forall Y \in \mathbf{R}^{n \times\left(n_{c}+m\right)}\right\}$, where the control matrices of the nominal $\mathrm{DH}_{g}$, parameterized by $A_{g}$ and $B_{g}$, and the current $D H$ systems are those of the parameterization (25c) of (25a). Then, the following properties hold:

(i) The system $D H$ is controllable if and only if $C(A, B)$ is full rank. If $C(A, B)$ is not full rank then there exists a control sequence such that the system $H$ is approximately controllable with state targeting error $0\left[\max \left(\left\|A-A_{g}\right\|_{2},\left\|B-B_{g}\right\|_{2}\right)\right]$.

(ii) The system $H$ is controllable if and only if $\operatorname{rank} C(A, B)=2 n$ and $\operatorname{rank} C\left(A_{c}, B_{c}\right)=n$. and

(iii) The system DH is controllable if rank $C\left(A_{g}, B_{g}\right)=2 n$

$$
\begin{aligned}
& \left\|A-A_{g}\right\|_{2}+\left\|B-A_{g}\right\|_{2} \\
& <\sqrt{\sup _{z \in \mathrm{Sp} A_{g}}\left\|\left[z I_{2 n}-A_{g} \vdots B_{g}\right]\right\|_{2}^{2}+\frac{1}{\sup _{z \in \mathrm{Sp} A_{g}}\left\|\left[z I_{2 n}-A_{g} \vdots B_{g}\right]\right\|_{2}}} \\
& \quad-\sup _{z \in \mathrm{Sp}_{g}}\left\|\left[z I_{2 n}-A_{g} \vdots B_{g}\right]\right\|_{2} .
\end{aligned}
$$

The system $H$ is controllable if, furthermore, $\operatorname{rank} C\left(A_{c}, B_{c}\right)=$ $n$.

(iv) The system DH is reachable if it is controllable and, furthermore, $A$ is nonsingular (in particular, if Property (iii) holds and, furthermore, $A_{g}$ is nonsingular and $\left\|A-A_{g}\right\|_{2}<$ $\left.1 /\left\|A_{g}^{-1}\right\|_{2}=\lambda_{\min }^{1 / 2}\left(A_{g} A_{g}^{T}\right)\right)$. The system $H$ is reachable if $D H$ is reachable and $A_{c}$ is nonsingular.

Proof. One gets by direct recursive calculation from (25a)

$x[k+i]$

$$
=A_{g}^{i} x[k]+\sum_{j=0}^{i-1} A_{g}^{i-j-1}\left(B_{g} g[k+j]+\delta[k+j]\right),
$$

$$
\begin{aligned}
\tilde{x}[k+2 n] & \\
= & x[k+2 n]-A_{g}^{2 n} x[k] \\
& -\sum_{j=0}^{2 n-1} A_{g}^{2 n-j-1}\left(A-A_{g}\right) x[k+j] \\
& -\sum_{j=0}^{2 n-1} A_{g}^{2 n-j-1}\left(B-B_{g}\right) g[k+j] \\
= & {\left[B_{g} \vdots A_{g} B_{g} \vdots \ldots A_{g}^{2 n-1} B_{g}\right]\left[\begin{array}{c}
g[k+2 n-1] \\
g[k+2 n-2] \\
\vdots \\
g[k]
\end{array}\right] } \\
= & C\left(A_{g}, B_{g}\right) C^{T}\left(A_{g}, B_{g}\right) g_{0}[k]
\end{aligned}
$$

provided that the input is generated from

$$
\begin{gathered}
\left(g^{T}[k+2 n-1], g^{T}[k+2 n-2], \ldots, g^{T}[k]\right)^{T} \\
=\left(\left[g_{c}^{T}[k+2 n-1], u^{T}[k+2 n-1]\right], \ldots,\right. \\
\left.\left[g_{c}^{T}[k], u^{T}[k]\right]\right)^{T}=C^{T}\left(A_{g}, B_{g}\right) g_{0}[k]=C^{T}\left(A_{g},\right. \\
\left.B_{g}\right)\left[C\left(A_{g}, B_{g}\right) C^{T}\left(A_{g}, B_{g}\right)\right]^{-1} \tilde{x}[k+2 n], \\
u((k+i) T+\tau)=U B_{c}^{T} e^{A_{c}^{T}(T-\tau)} g_{c}[k+i] \\
\text { for } \tau \in(0, T) ; i=0,1, \ldots, 2 n-1
\end{gathered}
$$


for $g_{0}[k] \in \mathbf{R}$, where $C(A, B)=\left[B \vdots A B \vdots \cdots \vdots A^{2 n-1} B\right]$ is the controllability matrix of the pair $(A, B)$. Note that since $\left(A_{g}, B_{g}\right)$ is controllable, then $C\left(A_{g}, B_{g}\right) C^{T}\left(A_{g}, B_{g}\right)$ is nonsingular. If $(A, B)$ is controllable, then $C(A, B)$ is full rank and fix $B_{g}=B$ so that $C\left(A_{g}, B-B_{g}\right)=C\left(A_{g}, A-A_{g}\right)=0$ and the sufficiency part of the result follows by choosing a control law (29a) and (29b) subject to

$$
\begin{array}{r}
g_{0}[k]=\left[C\left(A_{g}, B_{b}\right) C^{T}\left(A_{g}, B_{g}\right)\right]^{-1}(x[k+2 n] \\
\left.-A_{g}^{2 n} x[k]-\sum_{j=0}^{2 n-1} A_{g}^{2 n-j-1}\left(A-A_{g}\right) x[k+j]\right)
\end{array}
$$

and the system $H$ is then controllable. This proves the sufficiency part. The necessity part follows from (28) written in the equivalent form:

$$
x[k+2 n]-A_{g}^{2 n} x[k]=C(A, B)\left[\begin{array}{c}
g[k+2 n-1] \\
g[k+2 n-2] \\
\vdots \\
g[k]
\end{array}\right] .
$$

If $\operatorname{rank} C(A, B)<2 n$, then, given $x[k]$, there exists $\bar{x}^{*}$ such that $\operatorname{rank}\left[C(A, B), \bar{x}^{*}\right]>\operatorname{rank} C(A, B)$ with $x[k+2 n]=x^{*}$ and $x[k+2 n]-A_{g}^{2 n} x[k]=\bar{x}^{*}$ for some $x^{*} \in \mathbf{R}^{2 n}$. Thus, the following linear algebraic system of equations

$$
\bar{x}^{*}=C(A, B)\left[\begin{array}{c}
g[k+2 n-1] \\
g[k+2 n-2] \\
\vdots \\
g[k]
\end{array}\right]
$$

resulting from (31) is an incompatible one from RouchéFroebenius theorem of Linear Algebra. This leads to the proof of the necessity part of the first part of Property (i). Then, system $D H$ is controllable if and only if $C(A, B)$ is full rank. On the other hand, if the pair $(A, B)$ is not controllable while the pair $\left(A_{g}, B_{g}\right)$ is controllable, the system $D H$ is approximately controllable with state targeting error $-C\left(A_{g}, B-B_{g}\right) C^{T}\left(A_{g}, B_{g}\right) g_{0}[k]=0\left(\left\|A-A_{g}\right\|_{2},\left\|B-B_{g}\right\|_{2}\right)$. Property (i) has been proved. On the other hand, note from (la) that

$$
\begin{gathered}
x_{c}(k T+\tau)-\left(\int_{0}^{\tau} e^{A_{c}(\tau-\sigma)} d \sigma\right) \vartheta[k]-e^{A_{c} T} x_{c}[k] \\
=\int_{0}^{\tau} e^{A_{c}(\tau-\sigma)} B_{c} u(k T+\sigma) d \sigma, \\
\vartheta[k]=A_{c s} x_{c}[k]+A_{c d} x_{d}[k]+B_{c s} u[k],
\end{gathered}
$$

$\forall \tau \in[0, T), \forall k \in \mathbf{Z}_{+}$. If $D H$ is controllable, then $\operatorname{rank} C\left(A_{g}, B\right)=2 n$ and $x_{c}[k]$ and $x_{d}[k]$ are arbitrarily prefixed to $x_{c}^{*}[k]$ and $x_{d}^{*}[k]$ under some control sequence $u[k]$ so that $\vartheta[k]$ is prefixed as well to $\vartheta^{*}[k]=\vartheta^{*}\left[x^{*}[k]\right]$. Thus, from (33a) and (33b), $x_{c}(k T+\tau)$ is prefixed to any $x_{c}^{*}(k T+\tau), \forall \tau \in(0, T)$ and any given $k \in \mathbf{Z}_{+}$by a control law:

$$
\begin{aligned}
& u(k T+\sigma)=\int_{0}^{\tau} e^{A_{c}(\tau-\sigma)} B_{c} u(k T+\sigma) g_{u}[k], \\
& g_{u}[k]=\left[\int_{0}^{\tau} e^{A_{c}(\tau-\sigma)} B_{c} \int_{0}^{\tau} e^{A_{c}(\tau-\sigma)} B_{c} u(k T+\sigma)\right]^{-1} \\
& \cdot\left[x_{c}^{*}(k T+\tau)-\left(\int_{0}^{\tau} e^{A_{c}(\tau-\sigma)} d \sigma\right) \vartheta^{*}[k]\right. \\
& \left.-e^{A_{c} T} x_{c}^{*}[k]\right]
\end{aligned}
$$

provided that $\operatorname{rank} C\left(A_{c}, B_{c}\right)=n$ and the system $H$ is controllable. The sufficiency part of Property (ii) has been proved. The necessity follows by contradiction. Assume that $\operatorname{rank} C(A, B)<2 n$. Then, from Property (i), $D H$ is not controllable so that $H$ is not controllable at sampling points. Therefore, the system $H$ is controllable only if $\operatorname{rank} C(A, B)=$ $2 n$. From (33a) and (33b), $H$ is controllable if $D H$ is controllable, so that $x[k]$ and $\vartheta[k]$ can be prefixed in (33a) and (33b) from the controllability of $H$, only if $\operatorname{rank} C\left(A_{c}, B_{c}\right)=n$.

To prove Property (iii), note that $D H$ is reachable if and only if $\operatorname{rank}\left[z I_{2 n}-A \vdots B\right]=2 n, \forall z \in \operatorname{Sp} A$ from the Szëgo-Kalman-Popov (or discrete Yakubovich-KalmanPopov) controllability test. Equivalently, $D H$ is reachable if and only if

$$
\begin{aligned}
& {\left[z I_{2 n}-A \vdots B\right]\left[\begin{array}{c}
z^{*} I_{2 n}-A^{T} \\
B^{T}
\end{array}\right]} \\
& \quad=\left[\left(z I_{2 n}-A_{g}\right)\left(z^{*} I_{2 n}-A_{g}^{T}\right)+B_{g} B_{g}^{T}\right]\left(I_{2 n}\right. \\
& \left.\quad+\left[\left(z I_{2 n}-A_{g}\right)\left(z^{*} I_{2 n}-A_{g}^{T}\right)+B_{g} B_{g}^{T}\right]^{-1} \widetilde{G}(z)\right)
\end{aligned}
$$

is nonsingular, $\forall z \in \mathrm{Sp} A$, where $\left[\left(z I_{2 n}-A_{g}\right)\left(z^{*} I_{2 n}-\right.\right.$ $\left.\left.A_{g}^{T}\right)+B_{g} B_{g}^{T}\right]$ is nonsingular, $\forall z \in \operatorname{Sp} A_{g}$, since $\left(A_{g}, B_{g}\right)$ is controllable, and

$$
\begin{aligned}
\widetilde{G}(z)= & \left(A_{g}-A\right)\left(A_{g}^{T}-A^{T}\right)+\left(B-B_{g}\right)\left(B^{T}-B_{g}^{T}\right) \\
& +\left(z I_{2 n}-A_{g}\right)\left(A_{g}^{T}-A^{T}\right) \\
& +\left(A_{g}-A\right)\left(z^{*} I_{2 n}-A_{g}^{T}\right)+B_{g}\left(B^{T}-B_{g}^{T}\right) \\
& +\left(B-B_{g}\right) B_{g}^{T}
\end{aligned}
$$

so that

$$
\begin{gathered}
\|\widetilde{G}(z)\|_{2} \leq\left\|\left[A_{g}-A \vdots B-B_{g}\right]\right\|_{2} \\
\cdot\left(\left\|\left[A_{g}-A \vdots B-B_{g}\right]\right\|_{2}\right.
\end{gathered}
$$




$$
\left.+2\left\|\left[z I_{2 n}-A_{g} \vdots B_{g}\right]\right\|_{2}\right) ; \quad \forall z \in \mathbf{C}
$$

and $\sup _{z \in \mathrm{Sp} A_{g}}\|\widetilde{G}(z)\|_{2} \leq a^{2}+2 a b$, where

$$
\begin{aligned}
& a=\left\|\left[A_{g}-A \vdots B-B_{g}\right]\right\|_{2} ; \\
& b=\sup _{z \in \operatorname{SpA}_{g}}\left\|\left[z I_{2 n}-A_{g} \vdots B_{g}\right]\right\|_{2}
\end{aligned}
$$

so that $D H$ is controllable from (36), since $\left[\left(z I_{2 n}-A_{g}\right)\left(z^{*} I_{2 n}-\right.\right.$ $\left.\left.A_{g}^{T}+\right)+B_{g} B_{g}^{T}\right]$ is nonsingular $\forall z \in \operatorname{Sp} A_{g}$, if

$$
1>\sup _{z \in \operatorname{Sp} A_{g}}\left[\left(z I_{2 n}-A_{g}\right)\left(z^{*} I_{2 n}-A_{g}^{T}+\right)+B_{g} B_{g}^{T}\right]^{-1}
$$

$$
\cdot \widetilde{G}(z)
$$

which is guaranteed if

$$
\begin{aligned}
& a(2 b+a)<\frac{1}{b} \\
&= \frac{1}{\left\|\left[\left(z I_{2 n}-A_{g}\right)\left(z^{*} I_{2 n}-A_{g}^{T}\right)+B_{g} B_{g}^{T}\right]\right\|_{2}} ; \\
& \forall z \in \operatorname{Sp} A_{g}
\end{aligned}
$$

since

$$
\begin{aligned}
& \left\|\left[\left(z I_{2 n}-A_{g}\right)\left(z^{*} I_{2 n}-A_{g}^{T}\right)+B_{g} B_{g}^{T}\right]^{-1}\right\|_{2} \\
& \geq \frac{1}{\left\|\left[\left(z I_{2 n}-A_{g}\right)\left(z^{*} I_{2 n}-A_{g}^{T}+\right)+B_{g} B_{g}^{T}\right]\right\|_{2}} ; \\
& \forall z \in \mathbf{C} .
\end{aligned}
$$

Condition (40) holds if $a \in\left[0, \sqrt{b^{2}+b^{-1}}-b\right.$ ). This guarantees that $\operatorname{rank} C(A, B)=2 n$ and $D H$ is controllable. Since $\operatorname{rank} C\left(A_{c}, B_{c}\right)=n$, the system $H$ is controllable from Property (ii). Property (iii) has been proved.

To prove Property (iv) note that reachability of the discrete $\mathrm{DH}$ is guaranteed from controllability to the origin and the nonsingularity of its matrix of dynamics $A$. Those conditions are guaranteed from the conditions of Property (iii) if $A_{g}$ is nonsingular and $\left\|A-A_{g}\right\|_{2}<1 /\left\|A_{g}^{-1}\right\|_{2}$ which guarantees that $A=A_{g}+\left(A-A_{g}\right)=A_{g}\left(I_{2 n}+A_{g}^{-1}\left(A-A_{g}\right)\right)$ is nonsingular. On the other hand, if $D H$ is reachable, then $H$ is reachable if $A_{c}$ is nonsingular, since the pair $\left(A_{c}, B_{c}\right)$ is controllable.

Note that if (26) is tested for $z \in\left(\operatorname{Sp} A_{g}\right) \cap\{z \in \mathbf{C}$ : $|z| \geq 1\}$ (i.e., for the unstable and critically stable modes of $A_{g}$ ), then it becomes a stabilizability test of the current $\mathrm{DH}$ provided that the nominal $\mathrm{DH}_{\mathrm{g}}$ is stabilizable. In other words, stabilizability is the property implying that any uncontrollable mode is asymptotically stable while any unstable or critically stable mode is controllable.

\section{The Kalman-Yakubovich-Popov Lemma}

The following technical result will be then used for deriving a simplified but useful version of the KYP-Lemma (see [8, 37] and references therein) for the given $D H$ system in the event that the output matrix is identity and the input-output interconnection matrix is zero.

Lemma 6. The following properties hold:

(i) Assume that $\left[\begin{array}{c}\left(e^{i \omega} I_{2 n}-A\right)^{-1} B \\ I_{2 m}\end{array}\right]^{*} M\left[\begin{array}{c}\left(e^{i \omega} I_{2 n}-A\right)^{-1} B \\ I_{2 m}\end{array}\right] \preceq 0$ for some $M=M^{T} \in \mathbf{R}^{(3 n+m) \times(3 n+m)}$ and all $\omega \in[0, \infty]$, where $A=A_{g}+\widetilde{A}_{g}$ and $B=B_{g}+\widetilde{B}_{g}$.

Assume also that the discrete transfer matrix $\widetilde{G}_{g}(z)=$ $\left(z I_{2 n}-A_{g}\right)^{-1} \widetilde{A}_{g}$ is strictly bounded real (SBR), that $\operatorname{rank} \widetilde{B}_{g}=$ $\operatorname{rank}\left(B_{g} \vdots \widetilde{B}_{g}\right)$, and that $I_{2 m}+K_{B}$ is nonsingular, where $K_{B}$ is a real $(m+n) \times(m+n)$ matrix such that $\widetilde{B}_{g}=K_{B} B_{g}$. Then, there exists $M_{g}=M_{g}^{T} \in \mathbf{R}^{(3 n+m) \times(3 n+m)}$ such that

$$
\left[\begin{array}{c}
\left(e^{i \omega} I_{2 n}-A_{g}\right)^{-1} B_{g} \\
I_{2 m}
\end{array}\right]^{*} M_{g}\left[\begin{array}{c}
\left(e^{i \omega} I_{2 n}-A_{g}\right)^{-1} B_{g} \\
I_{2 m}
\end{array}\right]
$$

$$
\leq 0 .
$$

(ii) Assume that $\left[\begin{array}{c}\left(e^{i \omega} I_{2 n}-A_{g}\right)-1 \\ I_{2 m}\end{array} B_{g}\right]^{*} M^{\prime}\left[\begin{array}{c}\left(e^{i \omega} I_{2 n}-A_{g}\right)^{-1} B_{g} \\ I_{2 m}\end{array}\right] \preceq 0$ for some $M^{\prime}=M^{\prime T} \in \mathbf{R}^{(3 n+m) \times(3 n+m)}$ and all $\omega \in[0, \infty]$, where $A_{g}=A-\widetilde{A}_{g}$ and $B_{g}=B-\widetilde{B}_{g}$ with $A \neq A_{g}$ or $B \neq B_{g}$.

Assume also that the discrete transfer matrix $\widetilde{G}(z)=\left(z I_{2 n}{ }^{-}\right.$ $A)^{-1} \widetilde{A}_{g}$ is $S B R$, that $\operatorname{rank} \widetilde{B}_{g}=\operatorname{rank}\left(B: \widetilde{B}_{g}\right)$, and that $I_{2 m}+K_{B_{g}}$ is nonsingular, where $K_{B g}$ is a real $(m+n) \times(m+n)$ matrix such that $\widetilde{B}_{g}=\left(I_{2 n}+K_{B_{g}}\right)^{-1} B_{g}=-K_{B g} B$. Then, there exists $M_{G}=M_{G}^{T} \in \mathbf{R}^{(3 n+m) \times(3 n+m)}$ such that

$$
\left[\begin{array}{c}
\left(e^{i \omega} I_{2 n}-A\right)^{-1} B \\
I_{2(n+m)}
\end{array}\right]^{*} M_{G}\left[\begin{array}{c}
\left(e^{i \omega} I_{2 n}-A\right)^{-1} B \\
I_{2(n+m)}
\end{array}\right] \preceq 0 .
$$

Proof. Note that there exists a real $(m+n) \times(m+n)$ matrix $K_{B}$ such that $\widetilde{B}_{g}=K_{B} B_{g}$, from Rouché-Froebenious theorem, since $\operatorname{rank} \widetilde{B}_{g}=\operatorname{rank}\left(B_{g} \vdots \widetilde{B}_{g}\right)$ and that $B_{g}=\left(I_{m+n}+K_{B}\right)^{-1} B$ since $I_{m+n}+K_{B}$ is nonsingular. Also, since $\left\|\widetilde{G}_{g}(z)\right\|_{\infty}=$ $\sup _{\omega \in \mathbf{R}_{+}}\left\|\widetilde{G}_{g}\left(e^{i \omega}\right)\right\|<1$,

$$
\begin{aligned}
& \left(e^{i \omega} I_{2 n}-A\right)^{-1} B \\
& =\left[\left(e^{i \omega} I_{2 n}-A_{g}\right)\left(I_{2 n}-\left(e^{i \omega} I_{2 n}-A_{g}\right)^{-1} \widetilde{A}_{g}\right)\right]^{-1} \\
& \cdot\left(I_{m+n}+K_{B}\right) B_{g} \\
& =\left[\widehat{L}^{-1}(i \omega)\left(I_{2 n}-\left(e^{i \omega} I_{2 n}-A_{g}\right)^{-1} \widetilde{A}_{g}\right)\right]^{-1} \\
& \cdot\left(e^{i \omega} I_{2 n}-A_{g}\right)^{-1} B_{g},
\end{aligned}
$$


where

$$
\begin{array}{r}
\widehat{L}(i \omega)=\left(e^{i \omega} I_{2 n}-A_{g}\right)^{-1}\left(I_{m+n}+K_{B}\right)\left(e^{i \omega} I_{2 n}-A_{g}\right) ; \\
\forall \omega \in[0, \infty]
\end{array}
$$

so that

$$
\begin{aligned}
& {\left[\begin{array}{c}
\left(e^{i \omega} I_{2 n}-A_{g}\right)^{-1} B_{g} \\
I_{m+n}
\end{array}\right]^{*}\left(-M_{g}\right)\left[\begin{array}{c}
\left(e^{i \omega} I_{2 n}-A_{g}\right)^{-1} B_{g} \\
I_{m+n}
\end{array}\right]} \\
& \geq\left[\begin{array}{c}
\left(e^{i \omega} I_{2 n}-A_{g}\right)^{-1} B_{g} \\
I_{m+n}
\end{array}\right]\left(-\widehat{M}_{g}(i \omega)\right) \\
& \cdot\left[\begin{array}{c}
\left(e^{i \omega} I_{2 n}-A_{g}\right)^{-1} B_{g} \\
I_{m+n}
\end{array}\right]=\left[\begin{array}{c}
\left(e^{i \omega} I_{2 n}-A\right)^{-1} B \\
I_{m+n}
\end{array}\right]^{*} \\
& \cdot(-M)\left[\begin{array}{c}
\left(e^{i \omega} I_{2 n}-A\right)^{-1} B \\
I_{m+n}
\end{array}\right] \geq 0,
\end{aligned}
$$

where $M_{g}=M_{g}^{T} \in \mathbf{R}^{(3 n+m) \times(3 n+m)}$ has bounded entries and it is defined by

$$
\begin{aligned}
M_{g} & =\text { Block Diag }\left[\left[\widehat{L}^{-1}(i \bar{\omega})\right.\right. \\
\cdot & \left.\left.\left(I_{2 n}-\left(e^{i \bar{\omega}} I_{2 n}-A_{g}\right)^{-1} \widetilde{A}_{g}\right)\right]^{-1} \vdots I_{3 n+m}\right]^{*} \\
& \times M \text { Block Diag }\left[\left[\widehat{L}^{-1}(i \bar{\omega})\right.\right. \\
& \left.\left.\cdot\left(I_{2 n}-\left(e^{i \bar{\omega}} I_{2 n}-A_{g}\right)^{-1} \widetilde{A}_{g}\right)\right]^{-1} \vdots I_{3 n+m}\right]
\end{aligned}
$$

for $\bar{\omega} \in W$, with $W \subset \mathbf{R}_{+}$being nonempty, and such that the first matrix inequality of (46) holds. Property (i) has been proved. Property (ii) follows directly from duality considerations.

Remarks 7. (1) Note that Lemma 6(i) does not require for A to be a convergent matrix (i.e., a stability matrix on the discrete framework) while $A_{g}$ has to be a convergent matrix. Conversely, Lemma 6(ii) does not require for $A_{g}$ to be a convergent matrix while $A$ is a convergent matrix.

(2) If $\widetilde{G}_{g}(z)=\left(z I_{2 n}-A_{g}\right)^{-1} \widetilde{A}_{g}$ and $\widetilde{G}(z)=\left(z I_{2 n}-A\right)^{-1} \widetilde{A}_{g}$ are SBR, then $A$ and $A_{g}$ are convergent matrices and the identities

$$
\begin{aligned}
& \left(z I_{2 n}-A_{g}\right)=\left(z I_{2 n}-A\right)\left(I_{2 n}+\left(z I_{2 n}-A\right)^{-1} \widetilde{A}_{g}\right) \\
& =\left(z I_{2 n}-A_{g}\right)\left(I_{2 n}-\left(z I_{2 n}-A_{g}\right)^{-1} \widetilde{A}_{g}\right) \\
& \cdot\left(I_{2 n}+\left(z I_{2 n}-A\right)^{-1} \widetilde{A}_{g}\right)
\end{aligned}
$$

lead to

$$
\begin{aligned}
& \left(I_{2 n}-\left(z I_{2 n}-A_{g}\right)^{-1} \widetilde{A}_{g}\right) \\
& \quad=\left(I_{2 n}+\left(z I_{2 n}-A\right)^{-1} \widetilde{A}_{g}\right)^{-1} .
\end{aligned}
$$

(3) The identities

$$
\begin{aligned}
\widetilde{G}(z) & =\left(z I_{2 n}-A_{g}-\widetilde{A}_{g}\right)^{-1} \widetilde{A}_{g} \\
& =\left(I_{2 n}-\widetilde{G}_{g}(z)\right)^{-1} \widetilde{G}_{g}(z), \\
\widetilde{G}_{g}(z) & =\left(z I_{2 n}-A+\widetilde{A}_{g}\right)^{-1} \widetilde{A}_{g} \\
& =\left(I_{2 n}+\widetilde{G}(z)\right)^{-1} \widetilde{G}(z)
\end{aligned}
$$

imply that $\|\widetilde{G}(z)\|_{\infty} \leq \varepsilon$ and $\left\|\widetilde{G}_{g}(z)\right\|_{\infty} \leq \varepsilon_{g}$ are both SBR if $\varepsilon_{g} \in[0,1 / 2)$ and $\varepsilon \in\left[\varepsilon_{g} /\left(1-\varepsilon_{g}\right), 1\right)$, or if $\varepsilon \in[0,1 / 2)$ and $\varepsilon_{g} \in[\varepsilon /(1-\varepsilon), 1)$, and either $A_{g}$ is convergent, resulting in $A$ to be convergent, or $A$ is convergent resulting in $A_{g}$ to be convergent.

(4) Since $A_{g}$ is convergent, then the condition $\widetilde{G}_{g}(z)=$ $\left(z I_{2 n}-A_{g}\right)^{-1} \widetilde{A}_{g}$ being SBR of Lemma 6(i) is equivalent to $\left\|\left(I_{2 n}-A_{g}\right)^{-1} \widetilde{A}_{g}\right\|_{2}<1$ in the case when $A_{g}$ and $\widetilde{A}_{g}$ are positive and guaranteed if $\left\|\widetilde{A}_{g}\right\|_{2}<1 /\left\|\left(I_{2 n}-A_{g}\right)^{-1}\right\|_{2}$. See Theorem 3 of [9] for general linear discrete positive systems and the discrete KYP-Lemma. A similar conclusion arises for $\widetilde{G}(z)=\left(z I_{2 n}-A\right)^{-1} \widetilde{A}_{g}$ being SBR of Lemma 6(ii) if $A$ and $\widetilde{A}_{g}$ are positive. Then, such a condition is equivalent to $\|\left(I_{2 n}-\right.$ $A)^{-1} \widetilde{A}_{g} \|_{2}<1$ and guaranteed if $\left\|\widetilde{A}_{g}\right\|_{2}<1 /\left\|\left(I_{2 n}-A\right)^{-1}\right\|_{2}$. Theorem 3 of [9] would also conclude that $\left(z I_{2 n}-A_{g}\right)^{-1} \widetilde{A}_{g}$ and $\left(z I_{2 n}-A\right)^{-1} \widetilde{A}_{g}$ are SBR if and only if there is a diagonal $X\left(\in \mathbf{R}_{+}^{n \times n}\right)>0$ such that, respectively,

$$
\begin{gathered}
\left(\begin{array}{cc}
X & 0 \\
0 & I_{n}
\end{array}\right)-\left(\begin{array}{cc}
A_{g} & \widetilde{A}_{g} \\
I_{n} & 0
\end{array}\right)^{*}\left(\begin{array}{cc}
X & 0 \\
0 & I_{n}
\end{array}\right)\left(\begin{array}{cc}
A_{g} & \widetilde{A}_{g} \\
I_{n} & 0
\end{array}\right)>0, \\
\left(\begin{array}{cc}
X & 0 \\
0 & I_{n}
\end{array}\right)-\left(\begin{array}{cc}
A & \widetilde{A}_{g} \\
I_{n} & 0
\end{array}\right)^{*}\left(\begin{array}{cc}
X & 0 \\
0 & I_{n}
\end{array}\right)\left(\begin{array}{cc}
A & \widetilde{A}_{g} \\
I_{n} & 0
\end{array}\right)>0 .
\end{gathered}
$$

Theorem 8. Assume the following:

(1) $B_{g} \in \mathbf{R}_{+}^{n \times 2 m}, A_{g} \in \mathbf{R}_{+}^{n \times n}$ is convergent, and $\widetilde{A}_{g}>-A_{g}$ with the pair $\left(A_{g}, B_{g}\right)$ being controllable.

(2) $\widetilde{G}(z)=\left(z I_{2 n}-A_{g}-\widetilde{A}_{g}\right)^{-1} \widetilde{A}_{g}$ is $S B R$, and $I_{2 m}+K_{B_{g}}$ is nonsingular, where $K_{B g}$ is a real $(m+n) \times(m+n)$ matrix such that $\widetilde{B}_{g}=\left(I_{2 n}+K_{B_{g}}\right)^{-1} B_{g}>-B_{g}$ subject to $\operatorname{rank} \widetilde{B}_{g}=$ $\operatorname{rank}\left(B_{g}+\widetilde{B}_{g} \vdots \widetilde{B}_{g}\right)$.

(3) Constraint (26) holds and there exists a matrix $M_{g}=$ $M_{g}^{T} \in \mathbf{R}^{(3 n+m) \times(3 n+m)}$, which is nonnegative in all entries except for the last $2 m$ diagonal elements, such that (42) holds for all $\omega \in[0, \infty]$.

Then, the following properties hold:

(i) The pair $(A, B)$ is controllable, where $A\left(\in \mathbf{R}_{+}^{2 n \times 2 n}\right)=$ $A_{g}+\widetilde{A}_{g}$ is convergent. Also, there exists $M_{G}=M_{G}^{T} \epsilon$ $\mathbf{R}^{(3 n+m) \times(3 n+m)}$, which is nonnegative in all entries except for the last $(m+n)$ diagonal elements, such that (43) holds. 
(ii) The following matrix inequalities hold which are, respectively, equivalent to (42) and (43):

$$
\begin{gathered}
{\left[\begin{array}{c}
\left(I_{2 n}-A_{g}\right)^{-1} B_{g} \\
I_{m+n}
\end{array}\right]^{T} M_{g}\left[\begin{array}{c}
\left(I_{2 n}-A_{g}\right)^{-1} B_{g} \\
I_{m+n}
\end{array}\right] \preceq 0} \\
{\left[\begin{array}{c}
\left(I_{2 n}-A\right)^{-1} B \\
I_{m+n}
\end{array}\right]^{T} M_{G}\left[\begin{array}{c}
\left(I_{2 n}-A\right)^{-1} B \\
I_{m+n}
\end{array}\right] \preceq 0 .}
\end{gathered}
$$
that

(iii) There exist diagonal matrices $P_{g} \geq 0$ and $P_{G} \geq 0$ such

$$
\begin{aligned}
M_{g}+M_{g 0} \preceq 0 ; & M_{g 0}=\left[\begin{array}{cc}
A_{g}^{T} P_{g} A_{g}-P_{g} & A_{g}^{T} P_{g} B_{g} \\
B_{g}^{T} P_{g} A_{g} & B_{g}^{T} P_{g} B_{g}
\end{array}\right], \\
M_{G}+M_{G 0} \preceq 0 ; & M_{G 0}=\left[\begin{array}{cc}
A^{T} P_{G} A-P_{G} & A^{T} P_{G} B \\
B^{T} P_{G} A & B^{T} P_{G} B
\end{array}\right] .
\end{aligned}
$$

(iv) There exist $x_{g}, p_{g} \geq 0, u_{g}>0$ and $x, p \geq 0, u>0$ which satisfy

$$
\begin{array}{r}
\left(A_{g}-I_{2 n}\right) x_{g}+B_{g} u_{g} \leq 0, \\
{\left[x_{g}^{T}, u_{g}^{T} \vdots p_{g}^{T}\right]\left[\begin{array}{c}
M_{g} \\
A_{g}-I_{2 n} \vdots B_{g}
\end{array}\right] \leq 0,} \\
\left(A-I_{2 n}\right) x+B u \leq 0, \\
{\left[x^{T}, u^{T} \vdots p^{T}\right]\left[\begin{array}{c}
M_{G} \\
A-I_{2 n} \vdots B
\end{array}\right] \leq 0 .}
\end{array}
$$

(v) Constraints (42) (for all $\omega \in[0, \infty]$ ), (52), (54), and (56) are mutually equivalent and, also, (43) (for all $\omega \in[0, \infty]),(53)$, (55), and (57) are mutually equivalent. Furthermore, (42) implies (53), (55), and (57).

(vi) Assume that $\|\widetilde{G}(z)\|_{\infty} \leq \varepsilon$ and $\left\|\widetilde{G}_{g}(z)\right\|_{\infty} \leq \varepsilon_{g}$ are both SBR if $\varepsilon_{g} \in[0,1 / 2)$ and $\varepsilon \in\left[\varepsilon_{g} /\left(1-\varepsilon_{g}\right), 1\right)$, or if $\varepsilon \in[0,1 / 2)$ and $\varepsilon_{g} \in[\varepsilon /(1-\varepsilon), 1)$.

Proof. The first part of Property (i) follows from the controllability of the pair $\left(A_{g}, B_{g}\right)$ and the constraint (26) [Theorem 5(iii)], which guarantees the controllability of the pair $(A, B)$ with $A\left(\epsilon \mathbf{R}_{+}^{2 n \times 2 n}\right)$ being convergent and $B(\epsilon$ $\left.\mathbf{R}_{+}^{2 n \times(m+n)}\right)$ from the two first assumptions since $\widetilde{G}(z)=\left(z I_{2 n}-\right.$ $A)^{-1} \widetilde{A}_{g}$ is SBR, and $B\left(\in \mathbf{R}_{+}^{2 n \times(m+n)}\right)=B_{g}+\widetilde{B}_{g}$ since $B_{g} \in$ $\mathbf{R}_{+}^{n \times(m+n)}$. The second part follows from Lemma 6(ii) which concludes that (42) implies (43).

To prove Property (ii), note that constraint (52) holds since the equivalent constraint (42) (see [8]) holds from the given assumptions, Lemma 6(ii), and Property (i), and (53) holds since the equivalent constraint (43) holds. In the same way, Properties [(iii)-(v)] follow as well since constraints (42), (52), (54), and (56) (respectively, constraints (43), (53),
(55), and (57)) are mutually equivalent [8], under the given assumptions, Lemma 6(ii), and Property (i).

Property (vi) follows since constraints (42), (52), (54), and (56) are equivalent to each other and constraints (43), (53), (55), and (57) are also equivalent to each other while (42) and (43) imply to each other since $\|\widetilde{G}(z)\|_{\infty} \leq \varepsilon$ and $\left\|\widetilde{G}_{g}(z)\right\|_{\infty} \leq \varepsilon_{g}$ are both SBR (see Remarks 7(3)) since $A_{g}$ and $A$ are convergent.

Remark 9. If the semidefiniteness matrix inequalities in Theorem 8 are replaced by definiteness counterparts then the equivalences hold without the controllability assumption of the pair $\left(A_{g}, B_{g}\right)$.

The following result is concerned with the positive realness of a discrete nominal transfer matrix of the extended discrete nominal $\mathrm{DH}_{g}$ which guarantees that of the transfer matrix of a parametrical disturbed $\mathrm{DH}_{\mathrm{q}}$ under a set of structured parametrical perturbations of the dynamics, output, control, and interconnection matrices. The result is based on the equivalence between the positive realness of a transfer matrix and the associated state-space realization, namely, the Positive Real Lemma, so-called alternatively KalmanSzëgo-Popov Lemma or KSP Lemma, being a discrete version of the KYP-Lemma and of those ones with the Discrete Positive Factorization Lemma (so-called alternatively Youla's Factorization Lemma)

Theorem 10. Assume that $p=m+n, B_{g}, C_{g}$, and $D_{g}$ are positive, $A_{g}$ is positive, and the transfer matrix $G_{1 g}(z)=$ $G_{g}(z)-(\gamma / 2) I_{p}$ is positive real for some real constant $\gamma \in \mathbf{R}_{+}$, where $G_{g}(z)=C_{g}\left(z I_{2 n}-A_{g}\right)^{-1} B_{g}+D_{g}$ is strictly positive real, and that the triple $\left(A_{g}, B_{g}, C_{g}\right)$ is controllable and observable. Assume that the parameterizing matrices of the DH (25a), (25b), (25c), and (25d) are subject to parametrical disturbances so that

$$
\begin{aligned}
& A=A_{g}+\widetilde{A} \\
& B=B_{g}+\widetilde{B} \\
& C=C_{g}+\widetilde{C} \\
& D=D_{g}+\widetilde{D}
\end{aligned}
$$

with the disturbance matrices being subject to $\widetilde{A} \geq-A_{g}, \widetilde{B} \geq$ $-B_{g}, \widetilde{C} \geq-C_{g}$, and $\widetilde{D} \geq-D_{g}$. Assume, furthermore, that $C\left(A_{g}, B_{g}\right) C^{T}\left(A_{g}, B_{g}\right)$ and $C(A, B) C^{T}(A, B)$ are monomial. Then, both $\mathrm{DH}_{g}$ and $\mathrm{DH}$ are positive while the following properties hold:

(i) The transfer matrix $G(z)=C\left(z I_{2 n}-A\right)^{-1} B+D$ is strictly positive real (then $A$ is convergent), $A, B, C, D>0$ and $G_{1}(z)=G(z)-\gamma \rho / 2$ is positive real if there exist matrices $\widetilde{K} \in \mathbf{R}^{q \times p}, \widetilde{L} \in \mathbf{R}^{2 n \times q}$, where $q$ is some arbitrary positive integer, $\widetilde{P}\left(>-P_{g}\right) \in \mathbf{R}^{2 n \times 2 n}$ (being diagonal), $\widetilde{Q}\left(>-Q_{g}\right) \in \mathbf{R}^{2 n \times 2 n}$ satisfying the following set of matrix relations:

$$
\begin{aligned}
\widetilde{Q} & =\widetilde{P}+L_{g} \widetilde{L}^{T}+\widetilde{L} L_{g}^{T}+\widetilde{L} \widetilde{L}^{T}-\left(A_{g}^{T} P_{g} \widetilde{A}+\widetilde{A}^{T} P_{g} A_{g}\right) \\
& \geq-\bar{Q}_{g}=-Q_{g}-L_{g} L_{g}^{T}-L_{g} \widetilde{L}^{T}-\widetilde{L} L_{g}^{T}-\widetilde{L} \widetilde{L}^{T},
\end{aligned}
$$




$$
\begin{aligned}
\widetilde{C} & =\left(B_{g}^{T} \widetilde{P}+\widetilde{B}^{T}\left(P_{g}+\widetilde{P}\right)\right) A_{g}+\left(B_{g}^{T}+\widetilde{B}^{T}\right)\left(P_{g}+\widetilde{P}\right) \\
& \cdot \widetilde{A}+\left(K_{g}^{T}+\widetilde{K}^{T}\right) \widetilde{L}^{T}+\widetilde{K}^{T} L_{g}^{T}, \\
\widetilde{D} & \geq B_{g}^{T}\left(P_{g}+\widetilde{P}\right) \widetilde{B}+K_{g}^{T} \widetilde{K}+\frac{1}{2}\left(B_{g}^{T} \widetilde{P} B_{g}+\widetilde{B}^{T} \widetilde{P} \widetilde{B}\right. \\
& \left.+\widetilde{B}^{T} P_{g} \widetilde{B}+\widetilde{K}^{T} \widetilde{K}-\gamma(1-\rho) I_{p}\right)
\end{aligned}
$$

for some $\rho \in(0,1]$ and the given $\gamma \in \mathbf{R}_{+}$, for some existing matrices $Q_{g}(>0) \in \mathbf{R}^{2 n \times 2 n}, P_{g}(>0$ diagonal $) \in \mathbf{R}^{2 n \times 2 n}, K_{g} \in$ $\mathbf{R}^{q \times p}, L_{g} \in \mathbf{R}^{2 n \times q}$ which satisfy the following set of matrix identities:

$$
\begin{aligned}
A_{g}^{T} P_{g} A_{g}-P_{g} & =-L_{g} L_{g}^{T}-Q_{g}, \\
B_{g}^{T} P_{g} A_{g}+K_{g}^{T} L_{g}^{T} & =C_{g}, \\
K_{g}^{T} K_{g} & =D_{g}+D_{g}^{T}-\gamma I_{p}-B_{g}^{T} P_{g} B_{g} .
\end{aligned}
$$

(ii) If $Q_{g} \geq 0, \widetilde{Q} \geq-\bar{Q}_{g}$, then $G_{g}(z)$ and $G(z)$ are positive real even if $A_{g}$ is critically stable.

(iii) If $Q_{g} \geq 0, \widetilde{Q}>-\bar{Q}_{g}$, then $G_{g}(z)$ is positive real, $G(z)$ is strictly positive real, and $G_{1}(z)$ is positive real even if $A_{g}$ is critically stable.

(iv) If $G_{1 g}(z)$, and then $G_{g}(z)$, (respectively, $G_{1}(z)$ and then $G(z)$ ) are positive real then they do not satisfy Lemma 6, and conversely, for $M_{g}=\left[\begin{array}{cc}0 & -C^{T} \\ -C & \gamma I_{p}-\left(D_{g}+D_{g}^{T}\right)\end{array}\right]$.

Proof. Note that $\left[C\left(A_{g}, B_{g}\right) C^{T}\left(A_{g}, B_{g}\right)\right]^{-1}$ and $[C(A$, $\left.B) C^{T}(A, B)\right]^{-1}$ are positive since $C\left(A_{g}, B_{g}\right) C^{T}\left(A_{g}, B_{g}\right)$ and $C(A, B) C^{T}(A, B)$ are monomial so that the sequences $\left\{g_{g c}[k]\right\}$ and $\left\{g_{c}[k]\right\}$ are nonnegative for any nonnegative control. Note also that from the conditions on the parameterizing matrices both extended discrete systems describing the given hybrid system are positive. Note that $A_{g}$ is at least critically stable although nonnecessarily convergent. Note also that if $\gamma>0$ then $G_{g}(z)$ is strictly positive real and if $\gamma=0$ then it is positive real if $\mathbf{A}$ is at least critically stable (rather than convergent) with eventual simple poles of positive semidefinite on $|z|=1$. Note that $\operatorname{Re}\left(G_{g}\left(e^{i \omega}\right)+\operatorname{Re} G_{g}^{T}\left(e^{-i \omega}\right)\right)-\gamma I_{p} \geq 0$ for any $\omega \in[0, \infty]$ since $G_{g}(z)-(\gamma / 2) I_{p}$ is positive real and $p=m+n$. From the equivalence between the Discrete Factorization Lemma and the Discrete Positive Real Lemma [38], there exist a positive definite real matrix $P_{g}$, which is diagonal since $A_{g}$ is positive and convergent [3], and real matrices $K_{g}, L_{g}$, and $Q_{g}>0$ such that the matrix relations (61) hold implying from the Discrete Positive Factorization Lemma that

$$
\widehat{M}_{g}=-\left(M_{g}+M_{g 0}\right)=\left[\begin{array}{cc}
L_{g} L_{g}^{T}+Q_{g} & L_{g} K_{g} \\
K_{g}^{T} L_{g}^{T} & K_{g}^{T} K_{g}
\end{array}\right] \geq 0,
$$

where

$$
\begin{gathered}
M_{g}=\left[\begin{array}{cc}
0 & -C^{T} \\
-C & \gamma I_{p}-\left(D_{g}+D_{g}^{T}\right)
\end{array}\right] ; \\
M_{g 0}=\left[\begin{array}{cc}
A_{g}^{T} P_{g} A_{g}-P_{g} & A_{g}^{T} P_{g} B_{g} \\
B_{g}^{T} P_{g} A_{g} & B_{g}^{T} P_{g} B_{g}
\end{array}\right]
\end{gathered}
$$

so that the following factorization holds:

$$
\begin{aligned}
\operatorname{Re} & \left(G_{1 g}\left(e^{i \omega}\right)+\operatorname{Re} G_{1 g}^{T}\left(e^{-i \omega}\right)\right) \\
& =\operatorname{Re}\left(G_{g}\left(e^{i \omega}\right)+\operatorname{Re} G_{g}^{T}\left(e^{-i \omega}\right)\right)-\gamma I_{p} \\
& =W_{1 g}^{T}\left(e^{-i \omega}\right) W_{1 g}\left(e^{i \omega}\right) \geq 0 ; \quad \forall \omega \in[0, \infty],
\end{aligned}
$$

where $W_{1 g}\left(e^{i \omega}\right)=K+L^{T}\left(e^{i \omega} I_{2 n}-A_{g}\right)^{-1} B_{g}$. Thus, by invoking similar arguments of the equivalence between both lemmas, $G(z)=C\left(z I_{2 n}-A\right)^{-1} B+D-(\gamma \rho / 2) I_{p}$ is positive real for some given $\rho \in(0,1]$, if and only if, there exist a diagonal positive definite real matrix $P$ and real matrices $K, L$, and $Q>$ 0 , subject to $\widetilde{P} \succ-P_{g}, \widetilde{Q} \geq-Q_{g}$, satisfying

$$
\begin{aligned}
& P=P_{g}+\widetilde{P} ; \\
& K=K_{g}+\widetilde{K} \\
& L=L_{g}+\widetilde{L} ; \\
& Q=Q_{g}+\widetilde{Q}
\end{aligned}
$$

such that the following matrix relations hold:

$$
\begin{aligned}
A^{T} P A-P & =-L L^{T}-Q, \\
B^{T} P A+K^{T} L^{T} & =C, \\
K^{T} K & =D+D^{T}-\gamma \rho I_{p}-B^{T} P B .
\end{aligned}
$$

Now, direct calculations show that (61) guarantee (68) if (59) hold under the given constraints while similar conditions to (63)-(65) hold by replacing $M_{g} \rightarrow M_{G}, \widehat{M}_{g} \rightarrow \widehat{M}_{G}=$ $-\left(M_{G}+M_{G 0}\right) \leq 0$ with the matrices being defined from their counterparts with the replacements $\gamma \rightarrow \gamma \rho, A_{g} \rightarrow A=$ $A_{g}+\widetilde{A}, B_{g} \rightarrow B=B_{g}+\widetilde{B}, C_{g} \rightarrow C=C_{g}+\widetilde{C}, D_{g} \rightarrow$ $D=D_{g}+\widetilde{D}, Q_{g} \rightarrow Q=Q_{g}+\widetilde{Q}, P_{g} \rightarrow P=P_{g}+\widetilde{P}, L_{g} \rightarrow$ $L=L_{g}+\widetilde{L}, K_{g} \rightarrow K=K_{g}+\widetilde{K}$. Property (i) has been proved. The proofs of Properties (ii)-(iii) are similar and they follow straightforwardly. The proof of Property (iv) follows by comparing $M_{g}$ in (64) with (54) and by noting that it has negative entries except the last $p=m+n$ diagonal entries because the entries of $C_{g} \neq 0$ are nonnegative since the system is positive. So, the state-space realizations of $G_{1 g}(z)$ and $G_{g}(z)$ do not fulfill Lemma 6 for $M_{g}$ which has negative off-diagonal entries. A similar conclusion follows for $G_{1}(z)$ and $G(z)$. 
Remark 11. (1) Note that in Theorem 10(ii) $A_{g}$ and $A$ can be critically stable, since $G_{g}(z), G_{1 g}(z), G(z)$, and $G_{1}(z)$ are positive real, so that they can eventually possess simple eigenvalues, such that the four resulting matrices $H\left(e^{i \omega}\right)+$ $H^{T}\left(e^{-i \omega}\right)$, with $H$ being any of the four above ones, have positive semidefinite residuals at such simple critical poles.

(2) Note that if the nominal extended discrete system $\mathrm{DH}_{\mathrm{g}}$ is positive and Theorem 10 holds, then the extended discrete system $D H$ is positive and it is also positive in the input-output positivity (or "passivity") sense of $[13,15]$ (see also [30]) since positive realness of transfer matrices is equivalent in the discrete-time domain to

$$
\begin{aligned}
\sum_{k=k_{0}}^{k_{1}} y^{T}[k] g[k]= & \xi\left(x\left[k_{1}+1\right]\right)-\xi\left(x\left[k_{0}\right]\right) \\
& +\sum_{k=k_{0}}^{k_{1}} \lambda(x[k], g[k])
\end{aligned}
$$

with $g[k]=\left(g_{c}^{T}[k], u^{T}[k]\right)^{T}$ and $\lambda\left(x_{k}, u_{k}\right) \geq 0$ on any discrete-time interval $\left[k_{0}, k_{1}\right]$ with $k_{0} \geq 0$. In particular,

$$
\begin{aligned}
& \sum_{k=k_{0}}^{k_{1}} y^{T}[k] g[k]=\xi\left(x\left[k_{1}+1\right]\right)-\xi\left(x\left[k_{0}\right]\right) \\
& +\sum_{k=k_{0}}^{k_{1}} \lambda(x[k], g[k])=\frac{1}{2}\left\{x [ k _ { 1 } + 1 ] ^ { T } ( P _ { g } + \widetilde { P } ) x \left[k_{1}\right.\right. \\
& +1]-x\left[k_{0}\right]^{T}\left(P_{g}+\widetilde{P}\right) x\left[k_{0}\right]+\sum_{k=k_{0}}^{k_{1}}\left[\begin{array}{c}
x[k] \\
g[k]
\end{array}\right]^{T} \\
& \cdot\left[\begin{array}{r}
\left(L_{g}+\widetilde{L}\right)\left(L_{g}^{T}+\widetilde{L}^{T}\right)+Q_{g}+\widetilde{Q} \quad\left(L_{g}+\widetilde{L}\right)\left(K_{g}+\widetilde{K}\right) \\
\left(K_{g}^{T}+\widetilde{K}^{T}\right)\left(L_{g}^{T}+\widetilde{L}^{T}\right) \quad\left(K_{g}^{T}+\widetilde{K}^{T}\right)\left(K_{g}+\widetilde{K}\right)
\end{array}\right] \\
& \left.\cdot\left[\begin{array}{l}
x[k] \\
g[k]
\end{array}\right]\right\} \geq-\frac{1}{2} x\left[k_{0}\right]^{T}\left(P_{g}+\widetilde{P}\right) x\left[k_{0}\right] \geq-\frac{1}{2} x\left[k_{0}\right]^{T} \\
& \cdot P_{a} x\left[k_{0}\right]
\end{aligned}
$$

for any integers $k_{0} \geq 0$ and $k_{1} \geq k_{0}$ with $P_{a}=P_{g}-|\widetilde{P}|$ and a close relation for the nominal $D H_{g}$ with $\widetilde{L}=0, \widetilde{K}=0, \widetilde{P}=$ $\widetilde{Q}=0$.

(3) Usually, the positive real and positive factorization lemmas are stated for minimal (i.e., simultaneously controllable and observable) state-space realizations in order to exclude from the analysis eventual unstable and critically stable (in the nonstrict positive realness case) zero-pole cancellations in the transfer matrices $[13,15]$. The intuitive reason is that the state-space realization is got as a minimal one from the given transfer matrix so that it does not give information about eventual cancellations removed from the transfer matrices and its implication in the statespace descriptions when dealing with the Continuous or Discrete Positive Real Lemmas or their equivalent Youla's Factorization Lemmas.
Theorem 10 states a characterization of the admissible structured perturbations for the dynamics, output, control, and interconnection matrices of a state-space realization associated with the discrete nominal positive real transfer matrix which guarantee that the perturbed system $D H$ being positive maintains the positivity and the positive realness property of the nominal $\mathrm{DH}_{\mathrm{g}}$. Based on the Discrete Positive Real Lemma without invoking the factorization result, we now establish a parallel result to be applicable for nonstructured parametrical disturbances at the expense of testing the positive definiteness of an associated matrix.

Theorem 12. Assume that the hypothesis of Theorem 10 holds for the parametrical disturbance matrices. Then, both $\mathrm{DH}_{g}$ and $D H$ are positive and the following properties hold:

(i) The transfer matrix $G(z)=C\left(z I_{2 n}-A\right)^{-1} B+D$ is strictly positive real (then $A$ is convergent), $A, B, C, D>0$, and $G_{1}(z)=G(z)-\gamma \rho / 2$ is positive real if there exist matrices $\widetilde{S} \in \mathbf{R}^{2 n \times p}, \widetilde{P}\left(>P_{g}\right) \in \mathbf{R}^{2 n \times 2 n}$ (being diagonal), $\widetilde{R} \in \mathbf{R}^{p \times p}, \widetilde{Q} \in$ $\mathbf{R}^{2 n \times 2 n}$ satisfying the following set of matrix relations:

$$
\begin{aligned}
\widetilde{Q}= & \widetilde{P}-\left(A_{g}^{T} P_{g} \widetilde{A}+\widetilde{A}^{T} P_{g} A_{g}\right)-\left(A_{g}^{T} \widetilde{P} \widetilde{A}+\widetilde{A}^{T} \widetilde{P} A_{g}\right) \\
& -A_{g}^{T} \widetilde{P} A_{g}-\widetilde{A}^{T} P_{g} \widetilde{A}-\widetilde{A}^{T} \widetilde{P} \widetilde{A} \geq-\bar{Q}_{g}, \\
\widetilde{C}= & \left(B_{g}^{T} \widetilde{P}+\widetilde{B}^{T}\left(P_{g}+\widetilde{P}\right)\right) A_{g} \\
& +\left(B_{g}^{T}+\widetilde{B}^{T}\right)\left(P_{g}+\widetilde{P}\right) \widetilde{A}+\widetilde{S}^{T}, \\
\widetilde{D} \geq & B_{g}^{T}\left(P_{g}+\widetilde{P}\right) \widetilde{B}+\widetilde{R} \\
& +\frac{1}{2}\left(B_{g}^{T} \widetilde{P} B_{g}+\widetilde{B}^{T} \widetilde{P} \widetilde{B}+\widetilde{B}^{T} P_{g} \widetilde{B}-\gamma(1-\rho) I_{p}\right)
\end{aligned}
$$

for some $\rho \in(0,1]$ and the given $\gamma \in \mathbf{R}_{+}$, for some existing matrices $Q_{g}(>0) \in \mathbf{R}^{2 n \times 2 n}, P_{g}(>0$ diagonal $) \in \mathbf{R}^{2 n \times 2 n}, S_{g} \in$ $\mathbf{R}^{2 n \times p}$ and $R_{g} \in \mathbf{R}^{p \times p}$, which satisfy the following set of matrix identities:

$$
\begin{aligned}
A_{g}^{T} P_{g} A_{g}-P_{g} & =-L_{g} L_{g}^{T}-Q_{g}, \\
B_{g}^{T} P_{g} A_{g}+S_{g}^{T} & =C_{g}, \\
R_{g} & =D_{g}+D_{g}^{T}-\gamma I_{p}-B_{g}^{T} P_{g} B_{g}
\end{aligned}
$$

provided that

$$
\begin{aligned}
& \widehat{M}_{1 g}=\left[\begin{array}{cc}
L_{g} L_{g}^{T}+Q_{g} & S_{g} \\
S_{g}^{T} & R_{g}
\end{array}\right] \succeq 0 ; \\
& \widetilde{\widetilde{M}}_{1 g}=\left[\begin{array}{ll}
\widetilde{Q} & \widetilde{S} \\
\widetilde{S}^{T} & \widetilde{R}
\end{array}\right] \succeq-\widehat{M}_{1 g} \text {. }
\end{aligned}
$$

Furthermore, DH satisfies (71): 


$$
\begin{aligned}
& \sum_{k=k_{0}}^{k_{1}} y^{T}[k] g[k]=\frac{1}{2}\left\{x\left[k_{1}+1\right]^{T}\left(P_{g}+\widetilde{P}\right) x\left[k_{1}+1\right]-x\left[k_{0}\right]^{T}\left(P_{g}+\widetilde{P}\right) x\left[k_{0}\right]\right. \\
& \left.\quad+\sum_{k=k_{0}}^{k_{1}}\left[\begin{array}{c}
x[k] \\
g[k]
\end{array}\right]^{T}\left[\begin{array}{cc}
\left(L_{g}+\widetilde{L}\right)\left(L_{g}^{T}+\widetilde{L}^{T}\right)+Q_{g}+\widetilde{Q} & \left(L_{g}+\widetilde{L}\right)\left(K_{g}+\widetilde{K}\right) \\
\left(K_{g}^{T}+\widetilde{K}^{T}\right)\left(L_{g}^{T}+\widetilde{L}^{T}\right) & \left(K_{g}^{T}+\widetilde{K}^{T}\right)\left(K_{g}+\widetilde{K}\right)
\end{array}\right]\left[\begin{array}{l}
x[k] \\
g[k]
\end{array}\right]\right\}-\frac{1}{2} x\left[k_{0}\right]^{T}\left(P_{g}+\widetilde{P}\right) x\left[k_{0}\right] \\
& \geq-\frac{1}{2} x\left[k_{0}\right]^{T} P_{a} x\left[k_{0}\right]
\end{aligned}
$$

for any integers $k_{0} \geq 0$ and $k_{1} \geq k_{0}$ with $P_{a}=P_{g}-|\widetilde{P}|$ and $a$ close relation is satisfied by the nominal $D H_{g}$ with $\widetilde{L}=0, \widetilde{K}=$ $0, \widetilde{P}=\widetilde{Q}=0$.

(ii) If $Q_{g} \geq 0, \widetilde{Q} \geq-\bar{Q}_{g}$, then $G_{g}(z)$ and $G(z)$ are positive real even if $A_{g}$ is critically stable.

(iii) If $Q_{g} \geq 0, \widetilde{Q}>-\bar{Q}_{g}$, then $G_{g}(z)$ is positive real, $G(z)$ is strictly positive real, and $G_{1}(z)$ is positive real even if $A_{g}$ is critically stable.

Proof. Note that (74)-(76) lead to $\widehat{M}_{1 G}=\widehat{M}_{1 g}+\widetilde{\widetilde{M}}_{1 g} \geq 0$ for the nominal discrete hybrid system if the parametrical disturbances satisfy (72). Then, both $\mathrm{DH}_{g}$ and $\mathrm{DH}$ are positive, have positive real transfer matrices, and satisfy the respective passivity conditions (77) (see (65) and Remark 11(1)).

Example 13. A particular system of the studied hybrid class with $n=4$ is now discussed with some of the parameters being fixed "a prior" while others are primarily left undetermined in order to find the needed positive realness conditions. Consider the following the hybrid system (1a), (1b), and (1c) with $n_{c}=n_{d}=2, m=p=1, T=0.01$,

$$
\begin{aligned}
A_{c} & =\left[\begin{array}{cc}
-1 & 1 \\
0 & -2
\end{array}\right] ; \\
B_{c} & =\left[\begin{array}{l}
b_{c 1} \\
b_{c 2}
\end{array}\right]=\left[\begin{array}{l}
0 \\
1
\end{array}\right] ; \\
C_{c} & =\left[\begin{array}{ll}
c_{c 1} & c_{c 2}
\end{array}\right] ; \\
D_{c} & =d_{c}, \\
A_{c s} & =\left[\begin{array}{ll}
0.5 & 0 \\
0.1 & 0.5
\end{array}\right] ; \\
A_{c d} & =\left[\begin{array}{cc}
1 & 0 \\
0.1 & 0.5
\end{array}\right] ; \\
A_{d s} & =0.0001 I_{4}, \\
A_{d} & =\left[\begin{array}{ll}
0.5 & 1 \\
0.4 & 0
\end{array}\right] ; \\
B_{d} & =\left[\begin{array}{l}
0.01 \\
0.1
\end{array}\right] ;
\end{aligned}
$$

$$
\begin{aligned}
& B_{c s}=\left[\begin{array}{l}
0.2 \\
0.1
\end{array}\right] \\
& C_{d}=c_{d}^{T}=(1,0) ; \\
& D_{d}=d_{d}=1
\end{aligned}
$$

which leads to the following matrices:

$$
\begin{aligned}
& C=\left[C_{c}+C_{c s} \vdots 1,0\right] ; \\
& D=d=d_{c}+1 .
\end{aligned}
$$

One gets directly that

$$
\begin{aligned}
\Phi_{c}(0.01) & =e^{A_{c} \times 0.01}=\left[\begin{array}{cc}
0.988019 & 0.009851 \\
0 & 0.990050
\end{array}\right] ; \\
\Gamma_{c}(0.01) & =\int_{0}^{0.01} e^{A_{c}(0.01-\tau)} d \tau \\
& =\left[\begin{array}{cc}
0.990066 & 0.004951 \\
0 & 0.995017
\end{array}\right], \\
A_{c}^{\prime} & =\left[\begin{array}{cc}
0.989014 & 0.009856 \\
0.000995017 & 0.991045
\end{array}\right] ; \\
A_{d}^{\prime} & =\left[\begin{array}{cc}
0.6520998 & 0.0999894 \\
0.0432332 & 0.216166
\end{array}\right], \\
B_{0} & =\left[\begin{array}{c}
0.146403 \\
0.0432332 \\
0.25 \\
0.4
\end{array}\right] .
\end{aligned}
$$

If $V=1$, note that $A_{c}$ is Hurwitz and Metzler and then $\Phi_{c}(0.01)$ is positive and convergent. If $d_{c} \geq 0, B_{c}>0$, and $C_{c}>0$, then both the continuous-time subsystem and its discretized version to any sampling period are positive dynamic 
systems. Also, $A_{d}$ is convergent. The transfer function of the uncoupled continuous-time subsystem is

$$
\begin{aligned}
G_{c}(s) & =C_{c}\left(s I_{2}-A_{c}\right)^{-1} B_{c}+d_{c} \\
& =\frac{\left(c_{c 1} b_{c 1}+c_{c 2} b_{c 2}\right) s+\gamma_{c}}{s^{2}+3 s+2}+d_{c} ; \\
\gamma_{c} & =2 c_{c 1} b_{c 1}+\left(c_{c 1}+c_{c 2}\right) b_{c 2},
\end{aligned}
$$

where $s$ is the Laplace transform argument. It can be easily checked that the continuous transfer function is positive real if $b_{c 1}=c_{c 2}=0 ; 0 \leq \gamma_{c}=c_{c 1} b_{c 2} \leq 3$ and $d_{c}>0$. If $b_{c 2}>0$ and $c_{c 1}>0$ with $b_{c 1}=c_{c 2}=0$, then the triple $\left(A_{c}, B_{c}, C_{c}\right)$ is controllable and observable. If, in addition, $\gamma_{c}>0$, then $G_{c}(s)$ is strictly positive real. If, furthermore, $d_{c}>0$ or $\gamma_{c}<3$, then $G_{c}(s)$ is strongly strictly positive real in the sense that $\operatorname{Re} G_{c}(i \omega) \geq d_{c}>0, \forall \omega \in[-\infty, \infty]$ [19]. We can choose $b_{c 1}=c_{c 2}=0, c_{c 1}=b_{c 2}>0, d_{c} \geq 0$.

On the other hand, note that the uncoupled continuoustime subsystem is a mathematical model for some wellknown linear dynamic systems as a damped mechanical system, or an RLC electric circuit, described by the differential equation:

$$
\begin{aligned}
& v^{\prime \prime}(t)+3 v^{\prime}(t)+2 v(t)=f(t) \\
& \quad=\left(c_{c 1} b_{c 2}+2 d_{c}\right) u(t)+d_{c}\left(u^{\prime \prime}(t)+3 u^{\prime}(t)\right)
\end{aligned}
$$

forced by a term $f(t)$ calculated from a primary control $u(t)$ which is everywhere piecewise continuous if $d_{c}=0$ and everywhere twice continuous-time differentiable with piecewise continuous second time-derivative if $d_{c}>0$. Note that if $\left\|A_{c s}\right\|_{2}<0.581976$, then $A_{c}$ being Hurwitz guarantees that $A_{c}^{\prime}$ is convergent from the fulfillment of the stability constraint $1>\left\|\Phi_{c}^{-1}(0.01) \Gamma_{c}(0.01)\right\|_{2}\left\|A_{c s}\right\|_{2}$. The transfer function of the digital subsystem is $G_{d}\left(z^{-1}\right)=$ $\left(1-0.25 z^{-1}\right) /\left(1-0.5 z^{-1}-0.4 z^{-2}\right)$, where $z$ is the discrete transfer function argument representing in the time delay a one-step advance operator $q$, and equivalently, $q^{-1}$ is a one-step delay operator formally equivalent to $z^{-1}$. It can be directly checked that the digital transfer function is strictly positive real with $\operatorname{Re} G_{d}\left(e^{i \omega}\right) \geq d_{d}=1$ for $\omega \epsilon$ $[0,2 \pi]$. The extended $D H$ has four stable eigenvalues, namely, $0.367879,0.135335,0.930074,-0.430074$ in the free coupling case, that is, if $A_{d s}=0, A_{c d}=0$. By using a similar reasoning to that guaranteeing that $A_{c}$ being Hurwitz implies that $A_{c}^{\prime}$ is convergent, one concludes that the system matrix $A$ of the $D H$ is convergent if the sufficiency constraint below holds:

$$
\begin{aligned}
& \left\|A-\operatorname{Block} \operatorname{Diag}\left(A_{c}^{\prime}, A_{d}\right)\right\|_{2} \\
& \leq \max \left(\left\|\Gamma_{c}(0.1)\right\|_{2}\left\|A_{c d}\right\|_{2},\left\|A_{d s}\right\|_{2}\right) \\
& \leq \max \left(0.632121\left\|A_{c d}\right\|_{2},\left\|A_{d s}\right\|_{2}\right) \\
& <\frac{1}{\left\|\operatorname{Block} \operatorname{Diag}\left(A_{c}^{\prime-1}, A_{d}^{-1}\right)\right\|_{2}}=0.7406,
\end{aligned}
$$

$$
\begin{aligned}
& A_{g} \\
& =\left[\begin{array}{cccc}
0.989014 & 0.009856 & 0.6520998 & 0.0999894 \\
0.000995017 & 0.991045 & 0.0432332 & 0.216166 \\
0.0001 & 0.0001 & 0.85 & 0.5 \\
0.0001 & 0.0001 & 0 & 0.9
\end{array}\right] \\
& B_{g}=\left[\begin{array}{ccc}
0.146403 & 0 & 0 \\
0.0432332 & 0 & 0.08241999 \\
0.25 & 0 & 0 \\
0.4 & 0 & 0
\end{array}\right]
\end{aligned}
$$

Now, the Discrete Positive Real Lemma holds by defining an extended output $\bar{y}[k]=\left(y[k], g_{c}^{T}[k]\right)^{T}=\left(y[k], u_{2}[k]\right.$, $\left.u_{3}[k]\right)^{T}$ equalizing the dimension of the input, that is, $\bar{p}=$ $m=3$ while incorporating two extra components to the output to obtain the extended output with the corresponding replacements $C_{g} \rightarrow \bar{C}_{g}, D_{g} \rightarrow \bar{D}_{g}$ in (74) leading to

$$
\begin{aligned}
& P_{g}=I_{4} ; \\
& L_{g}=0 \text {; } \\
& \bar{C}_{g}=\left[\begin{array}{cccc} 
& C_{g} & \\
0 & 0 & 0 & 0 \\
0 & 0 & 0 & 0
\end{array}\right]=\left[\begin{array}{cccc}
1+c_{c s 1} & c_{c s 2} & 1 & 0 \\
0 & 0 & 0 & 0 \\
0 & 0 & 0 & 0
\end{array}\right] ; \\
& \bar{D}_{g}=\left[\begin{array}{ccc}
d_{d} & 0 & 0 \\
0 & 1 & 0 \\
0 & 0 & 1
\end{array}\right] \text {, } \\
& P_{g}-A_{g}^{T} P_{g} A_{g} \geq 0.994093 I_{4}>0, \\
& S_{g}^{T}=\bar{C}_{g}-B_{g}^{T} A_{g} \\
& =\left[\begin{array}{cccc}
c_{c s 1}+0.855097 & c_{c s 2}-0.044354 & 0.690162 & -0.148984 \\
0 & 0 & 0 & 0 \\
-0.000082 & -0.0811681 & -0.003563 & -0.017816
\end{array}\right] \text {, } \\
& R_{g}=\left[\begin{array}{ccc}
2 d_{d}-\gamma-0.033403 & 0 & -0.003563 \\
0 & 2-\gamma & 0 \\
-0.003563 & 0 & 2-\gamma-0.006793
\end{array}\right] \\
& =\left[\begin{array}{ccc}
1.21 & 0 & -0.003563 \\
0 & 1.26793 & 0 \\
-0.003563 & 0 & 1.2
\end{array}\right] \text {. }
\end{aligned}
$$

Now, for $\gamma=0.73207, d_{d}=0.9877365$,

$$
\begin{aligned}
& Q_{g} \\
& =\left[\begin{array}{cccc}
0.0217762 & -0.019591 & -1.1996452 & -0.517061 \\
-0.001974 & 0.017794 & -0.08024 & -0.430494 \\
-1.19964524 & -0.08024 & -0.430494 & -0.875032 \\
-0.517061 & -0.430494 & -0.875032 & 0.189968
\end{array}\right] \text {, }
\end{aligned}
$$




$$
\widetilde{\widetilde{M}}_{1 g}=\left[\begin{array}{cc}
\widetilde{Q} & \widetilde{S} \\
\widetilde{S}^{T} & \widetilde{R}
\end{array}\right] \succeq-\widehat{M}_{1 g} .
$$

(i) Note that if $c_{s 1}=c_{s 2}=0, d_{d}=1, \gamma=1$, then

$$
\begin{aligned}
R_{g} & =\left[\begin{array}{ccc}
0.956597 & 0 & -0.003563 \\
0 & 1 & 0 \\
-0.003563 & 0 & 0.9932069
\end{array}\right], \\
\gamma & =0.956597 \\
d_{d} & =0.9732985
\end{aligned}
$$

The condition $\widehat{M}_{1 g}>0$ holds since $1>s_{g}=\left\|S_{g}\right\|_{2} /$ $\min \left[\lambda_{\text {min }}\left(P_{g}-A_{g}^{T} P_{g} A_{g}\right), \lambda_{\text {min }}\left(R_{g}\right)\right]$ with $\left\|S_{g}\right\|_{2}=1.109815$. The positive realness condition of $\bar{G}_{g}(z)=\operatorname{Block} \operatorname{Diag}\left(G_{g}(z)-\right.$ $\left.\gamma / 2,(1-\gamma / 2) I_{2}\right)$ is guaranteed, where $G_{g}(z)$ is the transfer function of the nominal $D H_{g}$, and is ensured from the Discrete Positive Real Lemma under nominal parametrical conditions of the state-space realization. See Theorem 12(i), Eqs. (74). If the above basic constraint holds with a margin $\widetilde{\varepsilon}>$ 0 under the form $1>s_{g}+\widetilde{\varepsilon}$, then one gets from (72) admissible parametrical disturbances which keep the positive realness and the Discrete Positive Real Lemma. Alternative results can be got from Theorem 10 based on the hybrid version of Youla's Factorization Lemma.

(ii) However, note that the positive realness of $\mathrm{DH}$ is not fulfilled if, for instance, $\gamma \leq 2 d_{d}-1$ and $R_{g}=$ $\left[\begin{array}{ccc}1.96656 & 0 & -0.003563 \\ 0 & 1 & 0 \\ -0.003563 & 0 & 0.9932069\end{array}\right]$ has two nonstable eigenvalues 1, 1.99933, then $\lambda_{\text {min }}\left(P_{g}-A_{g}^{T} P_{g} A_{g}\right)=1.93469, \lambda_{\text {min }}\left(R_{g}\right)=0.9932069$.

A direct extension of the two last theorems for the case when $p<m+n$ is the following.

Corollary 14. Assume that the hypotheses of Theorems 10 and 12 hold except that $p<m+n$. Then, both theorems still hold for any fictitious extended output of $2 m$ components defined by $\bar{y}[k]=\left[y^{T}[k] \vdots u^{-T}[k]\right]^{T}$, where $u^{-}[k]$ is any set of $2 m-$ $p$ input components, with the matrices $D_{p}$ and $D$ having an appropriate block submatrix with zero and unit entries.

The following alternative result to Theorems 10 and 12 follows with a direct proof, then omitted, for the case when the control input in-between sampling instants is generated directly from its value at sampling instants instead of from an independent discrete sequence.

Corollary 15. Assume that the $g_{g c}[k]=\Delta_{g} u[k]$ and $g_{c}[k]=$ $\Delta u[k]$ for the nominal $D H_{g}$ and current $D H$ for some given matrices $\Delta_{g}, \Delta \in \mathbf{R}_{+}^{m \times m}$ and that hypothesis of Theorem 10 holds with the dimensional change $p=m$ for inputs and outputs resulting in the modified nominal and current control matrices of order $2 n \times p$ to those defined in (25c):

$$
\begin{aligned}
& B_{g} \\
& =\left[\int_{0}^{T} e^{A_{g c}(T-\tau)}\left[B_{g c s}+B_{g c} V B_{g c}^{T} e^{A_{g c}^{T}(T-\tau)} \Delta_{g}\right] d \tau\right] ; \\
& B_{g d}
\end{aligned}
$$

$$
B=\left[\int_{0}^{T} e^{A_{c}(T-\tau)}\left[B_{c s}+B_{c} V B_{c}^{T} e^{A_{c}^{T}(T-\tau)} \Delta\right] d \tau\right] .
$$

Then, Theorems 10 and 12 hold.

\section{Hyperstability Results}

Hyperstability is a property associated with the nonnegativity for all time of the input-output energy for all nonlinear and time-varying output-feedback controller belonging to a certain class which satisfies Popov's type inequality for any bounded initial conditions [11-13, 15] and which is linked to the positive realness of the transfer matrix of the linear dynamics. Thus, such a property is not associated, and it should not be confused, with the positivity of the trajectory solutions for any nonnegative initial conditions and controls. Both properties can be simultaneously inherent to some systems under certain conditions. The following hyperstability theorem holds as linked to Theorems 10 and 12 under controls got from any feedback nonlinear time-varying controller within the class satisfying Popov's type inequality.

Theorem 16. Consider the discrete hybrid system $D H,(25 a)$, (25b), (25c), and (25d) driven by a sequence $\{g[k]\}$, with $p=$ $m+n$, and $A_{g}, B_{g}, C_{g}$, and $D_{g}$ being positive matrices, and assume the following:

(1) The sequence $\{g[k]\}$ is generated by feedback from any nonlinear and eventually time-varying device of the form $g[k]=-\varphi([y[k]], k)$ which satisfies the following Popov's inequality:

$$
\sum_{k=k_{0}}^{k_{1}} y^{T}[k] \varphi([y[k]], k) \geq-\gamma_{0}^{2}
$$

for some finite real number $\gamma_{0} \neq 0$ and any given integers $k_{0} \geq 0$ and $k_{1}>k_{0}$.

(2) The transfer matrix $G_{g 1}(z)=C_{g}\left(z I_{2 n}-A_{g}\right)^{-1} B_{g}+D_{g}-$ $(\gamma / 2) I_{p}$ is positive real for some real constant $\gamma \in \mathbf{R}_{+}$so that its state-space realization satisfies (74)-(76) (equivalently (61)) for some existing matrices $Q_{g}(\succeq 0) \in \mathbf{R}^{2 n \times 2 n}, P_{g}(>0$ diagonal $) \epsilon$ $\mathbf{R}^{2 n \times 2 n}, S_{g} \in \mathbf{R}^{2 n \times p}$, and $R_{g} \in \mathbf{R}^{p \times p}$ (equivalently with existing matrices $K_{g} \in \mathbf{R}^{q \times p}, L_{g} \in \mathbf{R}^{2 n \times q}$ ).

(3) The parametrical disturbances satisfy the matrix constraints (72) or, equivalently, (59) with $\widetilde{P} \geq-P_{g}$. Then, the following properties hold:

(i) The given class of controls keeps the hyperstability of the closed-loop DH from that of $\mathrm{DH}_{g}$ in the sense that $\left\{\|x[k]\|_{2}\right\}$ is uniformly bounded for any given initial condition $x[0]$ and any nonlinear eventually time-varying controller satisfying Popov's inequality (89). If, furthermore, $\left(A_{g}, B_{g}\right)$ is controllable and (26) holds, then $(A, B)$ is controllable and $\left\{\|g[k]\|_{2}\right\}$ is uniformly bounded.

(ii) If $\widehat{M}_{1 G}=\widehat{M}_{1 g}+\widetilde{\widetilde{M}}_{1 g}>0, \widetilde{Q} \geq-Q_{g}, \widetilde{R} \geq-R_{g}$, then $\{x[k]\} \rightarrow 0,\{u[k]\} \rightarrow 0$ (even if $(A, B)$ is not controllable) under the given class of controls so that the closed-loop system is asymptotically hyperstable. Equivalently, $G_{1 g}(z)=C_{g}\left(z I_{2 n}-\right.$ $\left.A_{g}\right)^{-1} B_{g}+D_{g}-(\gamma / 2) I_{p}$ is strictly positive real. 
(iii) Assume that $G_{1 g}(z)$ is strictly positive real for some $\gamma \in \mathbf{R}_{+}$. Then, the closed-loop DH keeps the asymptotic hyperstability property from that of the nominal $\mathrm{DH}_{g}$ in the sense that Property (i) holds and, furthermore, $\left\{\|x[k]\|_{2}\right\},\left\{\left\|g_{c}[k]\right\|_{2}\right\}$, and $\left\{\|u[k]\|_{2}\right\}$ converge asymptotically to zero for any given initial condition $x[0]$.

(iv) Assume that $\widehat{M}_{1 G} \geq 0$ with $Q_{g}>0$ and $\widetilde{Q} \geq$ $-Q_{g}$ (so that $G_{g 1}(z)$ is positive real). Assume also that either $K_{g}^{T} K_{g}>0$ or $\left(A_{g}, B_{g}\right)$ is stabilizable while, furthermore and correspondingly, either $K^{T} K>0$ (equivalently $R>0$ ) or $(A, B)$ is stabilizable. Then, Property (iii) still holds.

(v) Properties (i)-(iii) hold for Corollary $14(p<m+n)$ and Corollary $15(p=m)$ with the given modifications of the parameterizations.

Proof. One gets from (86) and (89)

$$
\begin{aligned}
& +\infty>\gamma_{0}^{2}+\frac{1}{2} x\left[k_{0}\right]^{T}\left(P_{g}+\widetilde{P}\right) x\left[k_{0}\right] \geq \sum_{k=k_{0}}^{k_{1}} y^{T}[k] g[k] \\
& =\frac{1}{2}\left\{x\left[k_{1}+1\right]^{T}\left(P_{g}+\widetilde{P}\right) x\left[k_{1}+1\right]+\sum_{k=k_{0}}^{k_{1}}\left[\begin{array}{l}
x[k] \\
g[k]
\end{array}\right]^{T}\right. \\
& \cdot\left[\begin{array}{cc}
\left(L_{g}+\widetilde{L}\right)\left(L_{g}^{T}+\widetilde{L}^{T}\right)+Q_{g}+\widetilde{Q} & \left(L_{g}+\widetilde{L}\right)\left(K_{g}+\widetilde{K}\right) \\
\left(K_{g}^{T}+\widetilde{K}^{T}\right)\left(L_{g}^{T}+\widetilde{L}^{T}\right) & \left(K_{g}^{T}+\widetilde{K}^{T}\right)\left(K_{g}+\widetilde{K}\right)
\end{array}\right] \\
& \left.\cdot\left[\begin{array}{c}
x[k] \\
g[k]
\end{array}\right]\right\} \\
& \geq \frac{1}{2} x\left[k_{1}+1\right]^{T}\left(P_{g}+\widetilde{P}\right) x\left[k_{1}+1\right]
\end{aligned}
$$

for any integers $k_{0} \geq 0$ and $k_{1}>k_{0}$ with the fourblock partitioned matrices of (90a) being at least positive semidefinite (see Theorems 10 and 12). Since $P_{g}+\widetilde{P}>0$, the sequence $\left\{\|x[k]\|_{2}\right\}$ is uniformly bounded for any given initial condition $x[0]$ and any nonlinear eventually time-varying controller satisfying Popov's inequality (89). Thus, the current closed-loop system $D H$ is hyperstable as it is the nominal one. If $\left(A_{g}, B_{g}\right)$ is controllable and (26) holds, then $(A, B)$ is controllable for the parameterizations defined in $(25 \mathrm{c})$ and then the uniform boundedness of $\left\{\|x[k]\|_{2}\right\}$ implies that of $\left\{\|g[k]\|_{2}\right\}$. Property (i) has been proved. Under the conditions of Property (ii), since $\widehat{M}_{1 g}>0$, so that $Q_{g}>0$ and $R_{g}>$ 0 (equivalently, $K_{g}^{T} K_{g}>0$ ), and $\widetilde{\widehat{M}}_{1 g} \geq-\widehat{M}_{1 g}$, so that $\widetilde{Q} \geq-\bar{Q}_{g}, \widetilde{R} \geq-R_{g}$, then $\widehat{M}_{1 G}=\widehat{M}_{1 g}+\widetilde{\widetilde{M}}_{1 g}>0$ and $P_{g}>0$ and $\widetilde{P} \geq-P_{g}$, then it follows from the boundedness of the second inequality of (89) for all $k_{1}>k_{0}$ that the sampled state and input of the nominal and current $D H$ converge asymptotically to zero without the need for any controllability assumption. This proves Property (ii). Under the additional conditions of Property (iii), $A$ is convergent and $\{x[k]\} \rightarrow 0$ from the first identity of (68) which is a discrete Lyapunov matrix equation. Note that (74)-(76) hold with $Q_{g}>0$ and $\widetilde{Q} \geq-\bar{Q}_{g}$ (from the strict positive realness condition) and $R_{g}>0$ as well as $R>0$ (since $\widetilde{R} \geq-R_{g}$ ) and furthermore, $K_{g}^{T} K_{g}>0$ and $K^{T} K>0$.
Otherwise, $\lim _{\omega \rightarrow \infty} \operatorname{Re}\left(G_{1 g}\left(e^{i \omega}\right)+G_{1 g}^{T}\left(e^{-i \omega}\right)\right)=0$ and the nominal $G_{1 g}(z)$ would not be strictly positive real as it would happen with the disturbed transfer matrix. This implies that $\{x[k]\} \rightarrow 0$ since otherwise $\lim _{k_{1} \rightarrow \infty} \sum_{k_{0}}^{k_{1}} x^{T}[k] Q x[k]$ would be infinity from the system positivity. A similar argument concludes that $\{g[k]\} \rightarrow 0$, since $K^{T} K>0$, without requiring a controllability condition, since any eventual zero/pole cancellation in the transfer matrix is necessarily strictly stable (since the transfer matrix $G_{1 g}(z)$ is strictly positive real) so that any eventual uncontrollable mode is asymptotically stable. This proves Property (iii). To prove Property (iv), note that if $\widehat{M}_{1 G} \geq 0$ with $Q_{g}>0$ and $\widetilde{Q} \geq-\bar{Q}_{g}$, then $G_{1 g}(z)$ is positive real. From the system positivity and the finite upperboundedness of (89), $\lim _{k_{1} \rightarrow \infty} \sum_{k_{0}}^{k_{1}} x^{T}[k]\left(Q_{g}+\widetilde{Q}\right) x[k]<\infty$ for any $\widetilde{Q} \geq-Q_{g}$ implying that $\{x[k]\} \rightarrow 0$ for any given finite $x[0]$. In the same way, $\lim _{k_{1} \rightarrow \infty} \sum_{k_{0}}^{k_{1}} g^{T}[k] K^{T} K g[k]<\infty$. If $K^{T} K>0$, equivalently $Q>0,\{g[k]\} \rightarrow 0$. Else, if the discrete pair $(A, B)$ associated with $D H$ is stabilizable, then there exists a matrix transformation $T$ such that $T^{-1} A T=$ $\left[\begin{array}{cc}A_{c c} & A_{c \bar{c}} \\ 0 & A_{\bar{c}}\end{array}\right], T^{-1} B=\left[\begin{array}{c}B_{c c} \\ 0\end{array}\right]$ such that $\left(A_{c c}, B_{c c}\right)$ is controllable and $A_{\bar{c}}$ (associated with uncontrollable but stable modes from the stabilizability assumption) is convergent. Therefore, the uncontrollable (but globally asymptotically stable) discrete substate of the transformed state sequence $\left\{x_{\overline{c c}}[k]\right\} \rightarrow 0$ while its forced controllable substate of dimension $n_{c c} \leq n=$ $n_{c}+n_{d}$ (the inequality being strict if there exists at least one uncontrollable mode) satisfies

$$
\begin{aligned}
& x_{c c f}[k] \\
& =\left[\begin{array}{lllll}
B_{c c} & A_{c c} B_{c c} & \cdots & A_{c c}^{n_{c c}-1} B_{c c}
\end{array}\right]\left[\begin{array}{c}
g\left[k-n_{c c}\right] \\
g\left[k-n_{c c}-1\right] \\
\vdots \\
g[k-1]
\end{array}\right]
\end{aligned}
$$

so that $\left\{x_{c c f}[k]\right\} \rightarrow 0$ (and the whole controllable state $\left\{x_{c c}[k]\right\} \rightarrow 0$ since its homogeneous response vanishes as discrete time tends to infinity) if and only if $\{g[k]\} \rightarrow 0$ since its associated controllability matrix is full rank. Thus, $\left\{x_{c c}[k]\right\} \rightarrow 0,\left\{x_{\bar{c} c}[k]\right\} \rightarrow 0$ since the state in the original coordinates satisfies $\{x[k]\} \rightarrow 0$ and then $\{g[k]\} \rightarrow 0$. Property (iv) has been proved. Property (v) follows directly from Properties (i)-(iii) and Corollaries 14-15.

Note that the assumption (3) of Theorem 16 implies that $G(z)$ is positive real in Property (i) and strictly positive real in Property (ii).

The solutions for any time satisfy the global stability properties proved in the following result.

Corollary 17. Assume that $\widehat{V}$ is such that the factorization $V=\widehat{V} \widehat{V}^{T}$ holds, the pair $\left(A_{c}, B_{c} \widehat{V}^{T}\right)$ is controllable, and all the hypotheses of Theorem 16(i) hold. Then, the current hybrid system $H$ is globally asymptotically stable for any bounded initial conditions under any continuous-time control in-between samples (25b). As a result, the resulting hybrid $\mathrm{SH}$ is 
hyperstable. If the additional conditions of Theorem 16(ii) also hold, then $\mathrm{H}$ is, furthermore, asymptotically hyperstable.

Proof. From Theorem 16(i), the sequences $\{x[k]\},\{u[k]\}$, and $\left\{g_{c}[k]\right\}$ are uniformly bounded for bounded initial conditions. If the continuous-time control is generated from (25b) and $\left(A_{c}, B_{c} \widehat{V}^{T}\right)$ is controllable, then $u(t)$ is bounded for any $t \in[k T,(k+1) T), \forall k \in \mathbf{Z}_{0+}$. Also, $x(t)$ is bounded with the proved bounded initial condition $x[k]$ for any $k \in \mathbf{Z}_{0+}$ since the intersample control is bounded and the control at sampling instants is also bounded. Property (i) has been proved. Property (ii) is direct since, in addition, $x[k] \rightarrow 0$, $u[k] \rightarrow 0$ as $k \rightarrow \infty$, and $g_{c}[k] \rightarrow 0$ as $k \rightarrow \infty$ implies $u(t) \rightarrow 0$ for $t \in(k T,(k+1) T)$ as $k \rightarrow \infty$ from $(25 b)$.

Theorem 16 implies under the controllability of $\left(A_{c}, B_{c}\right)$ that the hybrid system $H$ is globally stable as proved in the subsequent result provided that the control input is piecewise continuous on its definition domain. It does not require either the stability or the critical stability of $A_{c}$.

Corollary 18. Consider the hybrid system $H,(1 a),(1 b)$, and (1c), with $p=m+n$, and $A_{g}, B_{g}, C_{g}$, and $D_{g}$ being positive matrices so that the pair $\left(A_{c}, B_{c}\right)$ is controllable and that the controllability Gramian $\int_{0}^{T} e^{A_{c}(T-\tau)} B_{c} B_{c}^{T} e^{A_{c}^{T}(T-\tau)} d \tau$ is monomial. Assume also that $\left(A_{g}, B_{g}\right)$ is controllable, that (26) holds, that the control input $u$ is everywhere piecewise continuous on its definition domain, and that an input driving sequence $\{g[k]\}$ is generated by feedback from any nonlinear and eventually time-varying device of the form

$$
\begin{gathered}
g[k]=\left(u^{T}[k], g_{c}^{T}[k]\right)^{T}=-\varphi([y[k]], k), \\
g_{c}[k]=\left(\int_{0}^{T} e^{A_{c}(T-\tau)} B_{c} B_{c}^{T} e^{A_{c}^{T}(T-\tau)} d \tau\right)^{-1} \\
\cdot\left(\int_{0}^{T} e^{A_{c}(T-\tau)} B_{c} u(k T+\tau) d \tau\right)
\end{gathered}
$$

and satisfies Popov's inequality (89) for some finite real number $\gamma_{0} \neq 0$ and any given integers $k_{0} \geq 0$ and $k_{1}>k_{0}$. Assume also that the assumptions (2) and (3) of Theorem 16 hold.

Then, $\left\{\|x(t)\|_{2}\right\}$ and $\left\{\|u(t)\|_{2}\right\}$ are uniformly bounded for any bounded initial conditions so that the hybrid system $\mathrm{H}$ is globally stable.

Proof. Since $\int_{0}^{T} e^{A_{c}(T-\tau)} B_{c} B_{c}^{T} e^{A_{c}^{T}(T-\tau)} d \tau$ is monomial, the sequence $\left\{g_{c}[k]\right\}$ is nonnegative from (93) for any nonnegative input. Also, since $G_{1 g}(z)=C_{g}\left(z I_{2 n}-A_{g}\right)^{-1} B_{g}+$ $D_{g}-(\gamma / 2) I_{p}$ is strictly positive real from assumption (2) of Theorem 16 the closed-loop nominal and current discrete $\mathrm{DH}_{g}$ and $\mathrm{DH}$ are hyperstable from Theorem 16(i). Also, since the pair $\left(A_{c}, B_{c}\right)$ is controllable, (93) is well posed since $\left(z I_{2 n}-A_{g}\right)^{-1}$ exists since it is the inverse of the controllability Gramian on the time interval $[0, T]$. Equation (1a) has the following unique solution for given initial conditions and control input:

$$
\begin{aligned}
& x_{c}(k T+\sigma) \\
& =e^{A_{c} \sigma} x_{c}[k]
\end{aligned}
$$

$$
\begin{array}{r}
+\int_{0}^{\sigma} e^{A_{c}(T-\tau)}\left(B_{c} u(k T+\tau)+B_{c s} u[k]\right) d \tau \\
+\left(\int_{0}^{\sigma} e^{A_{c}(T-\tau)} d \tau\right)\left(A_{c s} x_{c}[k]+A_{c d} x_{d}[k]\right) ; \\
\forall \tau \in(0, T) ; \forall k \in \mathbf{Z}_{+} .
\end{array}
$$

From Theorem 16(i) the sequences $\left\{\|x[k]\|_{2}\right\},\left\{\left\|g_{c}[k]\right\|_{2}\right\}$, and $\left\{\|u[k]\|_{2}\right\}$ are uniformly bounded and $u(t)$ is bounded on $[k T,(k+1) T), \forall k \in \mathbf{Z}_{0+}$. Then, from (94), $x_{c}(t)$ is bounded as well for $t \in(k T,(k+1) T), \forall k \in \mathbf{Z}_{0+}$.

Example 19. Consider Example 13 with a choice of the auxiliary input satisfying $\left\{g_{c}[k]\right\} \rightarrow 0$ and under the given conditions guaranteeing that $\bar{G}_{g}(z)=\operatorname{Block} \operatorname{Diag}\left(G_{g}(z)-\right.$ $\left.\gamma / 2,(1-\gamma / 2) I_{2}\right)$ is positive real. From Corollary 18 , the closed-loop nominal system $H_{g}$ and the discrete $D H_{g}$ are positive and asymptotically hyperstable, that is, globally asymptotically stable for the class of nonlinear and eventually time-varying nonlinear controllers within the class satisfying inequality (89). Thus, $\{u[k]\} \rightarrow 0,\{y[k]\} \rightarrow 0,\{x[k]\} \rightarrow$ $0, x(t) \rightarrow 0, y(t) \rightarrow 0$, and $u(t) \rightarrow 0$ as $t \rightarrow \infty$ if $u(t)$ is generated from (25b) in the intersample time interval for any given initial conditions.

Remark 20. Note from (94) the important observation that Corollary 18 does not conclude that $x(t) \rightarrow 0$ as $t \rightarrow \infty$ for any piecewise control input $u(t)$, even if $A_{c}$ is a stability matrix and $\{x[k]\} \rightarrow 0$ and $\{u[k]\} \rightarrow 0$, which has been got under the stronger conditions of Theorem 16(ii). If $\{x[k]\} \rightarrow$ 0 and $\{u[k]\} \rightarrow 0$, then

$$
\begin{aligned}
& \lim _{k \rightarrow \infty}\left(x_{c}(k T+\sigma)-\int_{0}^{\sigma} e^{A_{c}(T-\tau)} B_{c} u(k T+\tau) d \tau\right) \\
& \quad=0 ; \quad \forall \sigma \in[0, T) .
\end{aligned}
$$

We now introduce the concept of asymptotic hyperstability in the mean in the sense that the system is globally stable and, furthermore, the input and output power (and then its inputoutput instantaneous power) converge asymptotically to zero except eventually for a set of time instants zero measure.

Theorem 21. Assume the following:

(1) The pair $\left(A_{c}, B_{c}\right)$ is controllable.

(2) The nonlinear and eventually time-varying controller $\varphi(y(t), t)$ is everywhere piecewise continuous with respect to $y$ and continuous with respect to $t$ in $\mathbf{R}_{+}^{p} \times \mathbf{R}_{+}$.

(3) There is a partition of each interval $[k T,(k+1) T)$ in a finite number $s_{k}$ of disjoint intervals of length $0<T_{a k} \leq$ $T$ such that the sequences $\left\{P_{k_{k}}\right\},\left\{Q_{k j_{k}}\right\}$, and $\left\{R_{k j_{k}}\right\}$ consist of positive definite elements for existing partitions of each interval $[k T,(k+1) T)$ in a finite number $s_{k}$ of disjoint intervals of length $0<T_{a k} \leq T$ such that $\varphi(y(t), t)$ is continuous on each interval $\left[k T+(\ell-1) T_{a k},(k+1) T+\ell T_{a k}\right)$ for $\ell=1,2, \ldots, s_{k}, \forall k \in \mathbf{Z}_{+}$.

Then, the closed-loop system $H$ is asymptotically hyperstable in the mean so that $y\left(t_{k}(\sigma)\right) \rightarrow 0$ and $u\left(t_{k}(\sigma)\right) \rightarrow 0$ as for $\left(t_{k}+\sigma\right) \in \bigcup_{k \in \mathbf{Z}_{+}} \bigcup_{1 \leq \ell_{k} \leq s_{k}}\left(k T+\left(\ell_{k}-1\right) T_{a k},(k+1) T+\ell_{k} T_{a k}\right)$ as $k \rightarrow \infty$. If $\varphi(0, t)=0, \forall t \in \mathbf{R}_{+}$, then $H$ is asymptotically hyperstable. 
Proof. Consider an auxiliary eventually time-varying sampling period $T_{a k}=T / s_{k}$ for the time interval $[k T,(k+1) T)=$ $\left[\sum_{j=0}^{k-1} s_{j} T_{a j}, \sum_{j=0}^{k-1} s_{j} T_{a j}+s_{k} T_{a k}\right)$ and $s_{k}\left(\in \mathbf{Z}_{+}\right) \geq 1, \forall k \in$ $\mathbf{Z}_{+}$, is chosen so that $u(t)$ is continuous on $\left[k T+\left(\ell_{k}-\right.\right.$ 1) $\left.T_{a k}, k T+\ell_{k} T_{a k}\right)$ for $\ell=1,2, \ldots, s_{k}, \forall k \in \mathbf{Z}_{+}$. Note that, in general, the nonunique choice of the eventually time-varying auxiliary sampling period subject to a maximum threshold period $T_{a k} \leq \bar{T}_{a} \leq T, \forall k \in \mathbf{Z}_{+}$, can be always made since as $\varphi(y(t), t)$ is assumed to be everywhere piecewise continuous with respect to $y$ and continuous with respect to $t$, with eventual bounded isolated discontinuities, then $u(t)=$ $-\varphi(y(t), t)$ has the same property. Thus, one gets directly from (1a) that the solution of the continuous-time substate from $\left[k T, k T+T_{a k}\right)$ to $[k T,(k+1) T)=\left[k T, k T+s_{k} T_{a}\right)$ with initial conditions at $k T, \forall k \in \mathbf{Z}_{+}$, is as follows:

$$
\begin{aligned}
& x_{c}\left(k T+\ell_{k} T_{a k}\right)=x_{c}\left(\sum_{j=0}^{k-1} s_{j} T_{a j}+\ell_{k} T_{a k}\right) \\
& =e^{A_{c} T_{a k}} x_{c}\left(\sum_{j=0}^{k-1} s_{j} T_{a j}+\left(\ell_{k}-1\right) T_{a k}\right) \\
& +\int_{0}^{T_{a k}} e^{A_{c}\left(T_{a k}-\tau\right)}\left[B _ { c } u \left(\sum_{j=0}^{k-1} s_{j} T_{a j}+\left(\ell_{k}-1\right) T_{a k}\right.\right. \\
& +\tau)+B_{c s} u[k] d \tau+\left(\int_{0}^{T_{a k}} e^{A_{c}\left(T_{a k}-\tau\right)} d \tau\right) \\
& +\left(A_{c s} x_{c}[k]+A_{c d} x_{d}[k]\right)=e^{A_{c} T_{a k}} x_{c}\left(k T+\left(\ell_{k}\right.\right. \\
& \left.-1) T_{a k}\right)+\left(\int_{0}^{T_{a k}} e^{A_{c}\left(T_{a k}-\tau\right)} d \tau\right)\left(A_{c s} x_{c}[k]\right. \\
& \left.+A_{c d} x_{d}[k]\right) \\
& +\left[T_{a k} e^{A_{c}\left(T_{a k}-\tau_{c k}\right)} B_{c} \vdots T_{a k} e^{A_{c}\left(T_{a k}-\tau_{d k}\right)} B_{c s}\right] \bar{u}[k]
\end{aligned}
$$

for $\ell_{k}=1,2, \ldots, s_{k}, \forall k \in \mathbf{Z}_{+}$, where

$$
\begin{gathered}
v_{c}\left(\ell_{k}-1, T_{a}\right)[k] \\
=\left(\int_{0}^{T_{a k}} e^{A_{c}\left(T_{a k}-\tau\right)} B_{c} B_{c}^{T} e^{A_{c}^{T}\left(T_{a k}-\tau\right)} d \tau\right)^{-1} \\
\cdot\left(\int _ { 0 } ^ { T _ { a k } } e ^ { A _ { c } ( T _ { a k } - \tau ) } B _ { c } u \left(\sum_{j=0}^{k-1} s_{j} T_{a j}+\left(\ell_{k}-1\right) T_{a k}\right.\right. \\
+\tau) d \tau), \\
\bar{u}[k]=\left[\begin{array}{c}
\left.u\left(\sum_{j=0}^{k-1} s_{j} T_{a j}+\left(\ell_{k}-1\right) T_{a k}+\tau_{c k}\right)\right] \\
u[k]
\end{array}\right]
\end{gathered}
$$

$\tau_{c k}=\tau_{c k}\left(\ell_{k}-1, T_{a k}\right)$ and $\tau_{d k}=\tau_{d k}\left(T_{a k}\right)$ are vector functions from $\left(0, T_{a k}\right)$ to $\mathbf{R}_{+}^{n}: \forall k \in \mathbf{Z}_{+}$after picking-up the values in the open intersample fictitious period searching its value leading to the integral mean value in the above identities, one per row of $e^{A_{c}\left(T_{a k}-\tau_{c k}\right)} B_{c}$ and one per row of $e^{A_{c}\left(T_{a k}-\tau_{d k}\right)} B_{c s}$ and the corresponding input components, $\forall k \in \mathbf{Z}_{+}$. The matrix inverse in (97), which is the inverse of the controllability Gramian on $\left[0, T_{a k}\right]$, exists since the pair $\left(A_{c}, B_{c}\right)$ of nonnegative matrices is controllable on any time interval $\left[0, T_{a k}\right]$ of nonzero measure. Thus, one gets from (96) that

$$
\begin{aligned}
& \lim _{k \rightarrow \infty}\left(x_{c}\left(\sum_{j=0}^{k-1} s_{j} T_{a j}+\ell_{k} T_{a k}\right)-e^{A_{c} T_{a k}} x_{c}\left(\sum_{j=0}^{k-1} s_{j} T_{a j}\right.\right. \\
& \left.+\left(\ell_{k}-1\right) T_{a k}\right)-T_{a k}\left[e^{A_{c}\left(T_{a k}-\tau_{c k}\right)} B_{c} u(k T\right. \\
& \left.\left.\left.+\left(\ell_{k}-1\right) T_{a k}+\tau_{c k}\right)\right]\right)=0, \\
& \lim _{k \rightarrow \infty}\left(y\left(\sum_{j=0}^{k-1} s_{j} T_{a j}+\ell_{k} T_{a k}\right)-C_{c} e^{A_{c} T_{a k}} x_{c}\left(\sum_{j=0}^{k-1} s_{j} T_{a j}\right.\right. \\
& \left.+\left(\ell_{k}-1\right) T_{a k}\right)-T_{a k}\left[C_{c} e^{A_{c}\left(T_{a k}-\tau_{c k}\right)} B_{c} u(k T\right. \\
& \left.\left.\left.+\left(\ell_{k}-1\right) T_{a k}+\tau_{c k}\right)\right]\right)-D_{c} u\left(\sum_{j=0}^{k-1} s_{j} T_{a j}\right. \\
& \left.+\ell_{k} T_{a k}\right)=0
\end{aligned}
$$

since $\{u[k]\} \rightarrow 0$ and $\{y[k]\} \rightarrow 0$. Thus, for any given $\varepsilon=$ $\left(\varepsilon_{1}, \varepsilon_{2}, \ldots, \varepsilon_{n_{c}}\right)^{T}>0$, there exists $t_{k_{0}}=t_{k_{0}}(\varepsilon) \in \mathbf{R}_{+}$such that, for any $k_{1}\left(>k_{0}\right) \in \mathbf{Z}_{+}$and associate $t_{k_{1}}>t_{k_{0}} \in \mathbf{R}_{+}$by defining the auxiliary sampling instants $t_{\sum_{j=0}^{k-1} s_{j}+\ell_{k}}$, one gets from (90a) and (90b)

$$
\begin{aligned}
+\infty & >\bar{\gamma}_{0}^{2}=\gamma_{0}^{2}+\frac{1}{2} x\left[t_{k_{0}}\right]^{T}\left(P_{g c k_{0}}+\widetilde{P}_{c k_{0}}\right) x\left[t_{k_{0}}\right] \\
\geq & \sum_{k=k_{0}}^{k_{1}} \sum_{\ell_{j_{k}}=0}^{s_{k}} y^{T}\left[t_{k}+\ell_{j_{k}} T_{a k}+\tau_{c j_{k}}\right] u\left[t_{k}+\ell_{j_{k}} T_{a k}+\tau_{c j_{k}}\right] \\
= & \frac{1}{2}\left\{x\left[t_{k_{1}+1}\right]^{T}\left(P_{g k_{1}}+\widetilde{P}_{k_{1}}\right) x\left[t_{k_{1}+1}\right]+\varepsilon\left(t_{k}\right)\right. \\
& +\sum_{k=k_{0}}^{k_{j_{j}}=0} \sum_{\ell_{k}}^{s_{k}}\left[\begin{array}{ll}
x\left[t_{k}+\ell_{j_{k}} T_{a k}+\tau_{c j_{k}}\right] \\
g\left[t_{k}+\ell_{j_{k}} T_{a k}+\tau_{c j_{k}}\right]
\end{array}\right]^{T} \\
& \left.\cdot\left[\begin{array}{cc}
L_{k j_{k}} L_{k j_{k}}^{T}+Q_{j_{k}} & L_{k j_{k}} \\
K_{k j_{k}}^{T} L_{k j_{k}}^{T} & K_{k j_{k}}^{T} K_{k j_{k}}
\end{array}\right]\left[\begin{array}{l}
x\left[t_{k}+\ell_{j_{k}} T_{a k}+\tau_{c j_{k}}\right] \\
g\left[t_{k}+\ell_{j_{k}} T_{a k}+\tau_{c j_{k}}\right]
\end{array}\right]\right\}
\end{aligned}
$$

with $\left|\varepsilon\left(t_{k}\right)\right| \leq \varepsilon$ for $k>k_{0}$, where $y_{c}(t)=C_{c} x_{c}(t)+D_{c} u(t)$ for $t \in[k T,(k+1) T)=\left[\sum_{j=0}^{k-1} s_{j} T_{a j}, \sum_{j=0}^{k-1} s_{j} T_{a j}+s_{k} T_{a k}\right)$ and 
$y_{c}\left[t_{k}\right]=y\left(\sum_{j=0}^{k-1} s_{j} T_{a j}\right)-y_{d}\left[\sum_{j=0}^{k-1} s_{j} T_{a j}\right], \forall k \in \mathbf{Z}_{+}$. Note from (100) that since $\varepsilon\left(t_{k}\right) \rightarrow 0$ as $k \rightarrow \infty$, since $\varepsilon$ can be taken to be arbitrarily small, and simultaneously since the closedloop $D H$ is asymptotically hyperstable since the matrices $Q_{g k}$, $P_{g k}, R_{g k}$ and $Q_{k}, P_{k}, R_{k}$, are positive definite matrices for all $k \in \mathbf{Z}_{+}$, then $\{x[k]\} \rightarrow 0,\{g[k]\} \rightarrow 0,\{u[k]\} \rightarrow 0,\{y[k]\} \rightarrow$ $0,\left\{\begin{array}{c}x\left[t_{k}+\ell_{j_{k}} T_{a k}+\tau_{c j_{k}}\right] \\ g\left[t_{k}+\ell_{j_{k}} T_{a k}+\tau_{c j_{k}}\right]\end{array}\right\} \rightarrow 0$, and $\left\{u\left[t_{k}+\ell_{j_{k}} T_{a k}+\tau_{c j_{k}}\right]\right\} \rightarrow 0$ as it follows from the direct extension Theorem 16(iv) to timevarying parameterizations under sufficiency type conditions of asymptotic hyperstability, provided that $q=p$ and $K_{k}$ is nonsingular for all $k \in \mathbf{Z}_{+}$[13]. Since the state, input, and output have nonnegative components, one also gets that $x_{c}(t) \rightarrow 0$ and $y(t) \rightarrow 0$ as $t \rightarrow \infty$ except, eventually, at isolated time instants where the nonlinearity $\varphi(y(t), t)$ is not continuous.

If the linear part of the system is not positive real, or even stable, or if it is suited to improve its relative stability, a linear feedback law can be injected prior to the operation by the nonlinear device towards the achievement of positive realness or strictly positive realness of the transfer matrix describing the linear feed-forward block. In particular, assume that following state-feedback linear control law is given:

$$
\begin{aligned}
& u(t)=F_{c} x_{c}(t)+G_{c} r(t), \\
& u[k]=F_{d c} x_{c}[k]+F_{d d} x_{d}[k]+G_{d} r[k] ;
\end{aligned}
$$

$\forall t \in[k T,(k+1) T) ; \forall k \in \mathbf{Z}_{+}$, where the various matrices are of the appropriate orders and nonnegative entries with $r(t)=-\varphi(y(t), t)$ being an outer reference control generated via output-feedback by nonlinear and, eventually, a timevarying nonlinearity in the form $\varphi(y(t), t)$ subject to Popov's type inequality. If the control law is replaced in (1a)-(1c), then the closed-loop hybrid system $H$ becomes $H_{c l}$ described according to the following parametrical replacements:

$$
\begin{aligned}
& A_{c} \longrightarrow \bar{A}_{c}=A_{c}+B_{c} F_{c c} \\
& A_{c s} \longrightarrow \bar{A}_{c s}=A_{c s}+B_{c s} F_{d c} \\
& A_{c d} \longrightarrow \bar{A}_{c d}=A_{c d}+B_{c s} F_{d d}+B_{c} F_{c d}, \\
& B_{c} \longrightarrow \bar{B}_{c}=B_{c} G_{c} \text {; } \\
& B_{c s} \longrightarrow \bar{B}_{c s}=B_{c s} G_{d} \text {; } \\
& B_{d} \longrightarrow \bar{B}_{d}=B_{d} G_{d} \\
& A_{d s} \longrightarrow \bar{A}_{d s}=A_{d s}+B_{d} F_{d c} \\
& A_{d} \longrightarrow \bar{A}_{d}=A_{d}+B_{d} F_{d d} \\
& C_{c} \longrightarrow \bar{C}_{c}=C_{c}+D_{c} F_{c c} \\
& C_{c s} \longrightarrow \bar{C}_{c s}=C_{c s}+D_{d} F_{d c}, \\
& C_{d} \longrightarrow \bar{C}_{d}=C_{d}+D_{d} F_{d d} \text {; } \\
& D_{c} \longrightarrow \bar{D}_{c}=D_{c} G_{d} \text {; } \\
& D_{d} \longrightarrow \bar{D}_{d}=D_{d} G_{d} \text {. }
\end{aligned}
$$

Consequently, (3) to (6) are modified, as driven by $r(t)$, according to

$$
\begin{aligned}
A \longrightarrow \bar{A} & =\left[\begin{array}{c}
e^{\bar{A}_{c} T}\left(I_{n_{c}}+\left(\int_{0}^{T} e^{-\bar{A}_{c} \tau} d \tau\right) \bar{A}_{c s}\right) e^{\bar{A}_{c} T}\left(\int_{0}^{T} e^{-\bar{A}_{c} \tau} d \tau\right) \bar{A}_{c d} \\
\bar{A}_{d}
\end{array}\right] \\
B_{0} \longrightarrow \bar{B}_{0} & =\left[\begin{array}{c}
\left.e^{\bar{A}_{c} T}\left(\int_{0}^{T} e^{-\bar{A}_{c} \tau} d \tau\right) \bar{B}_{c s}\right] \\
\bar{B}_{d}
\end{array}\right] \\
B_{v} \longrightarrow \bar{B}_{v} & =\left[\begin{array}{cc}
\bar{B}_{0} & I_{n_{c}} \\
& 0_{n_{d} \times n_{d}}
\end{array}\right] \\
C \longrightarrow \bar{C} & =\left[\begin{array}{l}
\bar{C}_{c}+\bar{C}_{c s} \vdots \bar{C}_{d}
\end{array}\right] \\
\bar{D} & =\bar{D}_{c}+\bar{D}_{d} .
\end{aligned}
$$

The following result is related to the achievement of the hyperstability of the closed-loop extended discrete hybrid system under the given control law as well as the asymptotic hyperstability in the mean of $H_{c \ell}$.
Theorem 22. Assume that $\left(A_{c}, B_{c}\right),\left(A_{d}, B_{d}\right),\left(A_{d s}, B_{d}\right)$, and $\left(A_{c d}, B_{c}\right)$ and $\left(A_{c d}, B_{c s}\right)$ are controllable pairs. Then, an appropriate feasible parameterization of the control gains in the fictitious discrete control law (92)-(93), with the replacement 
$u(\cdot) \rightarrow r(\cdot)$ and the system closed-loop reparameterization (103), associated with the feedback control law (101), may lead to a positive real transfer matrix of the positive closed-loop system $D_{c e}$ and then its hyperstability if $r(t)=-\varphi(y(t), t)$ for any nonlinear and eventually time-varying nonlinearity $\varphi(y(t), t)$ which satisfies Popov's type inequality.

If, furthermore, $\mathrm{DH}_{\mathrm{cl}}$ is asymptotically hyperstable and the assumptions (2) and (3) of Theorem 21 hold, then the closedloop hybrid $\mathrm{H}_{c e}$ is asymptotically hyperstable in the mean.

Proof. We refer with superscript bars to any matrices for either the parameterization or the Positivity Real Lemma $(\bar{P}$, $\bar{Q}, \bar{L}, \bar{S}$, and $\bar{R}$ ) after performing the control law (101). Note that, under controllability of any pair $(A, B)$, it is possible to choose a state-feedback control gain $X$ for the achievement of any given arbitrarily prescribed stable closed-loop placement. Since $\left(A_{c}, B_{c}\right),\left(A_{d}, B_{d}\right),\left(A_{d s}, B_{d}\right)$, and $\left(A_{c d}, B_{c}\right)$ and $\left(A_{c d}, B_{c s}\right)$, so $\left(A_{c d}+B_{c s} F_{d d}, B_{c}\right)$, are controllable pairs, it is feasible to choose the control gains $F_{c c}$ and $F_{d d}$ in such a way that $\bar{A}_{c}$, and then $e^{\bar{A}_{c} T}$, and $\bar{A}_{d}$ have stable eigenvalues being as largely dominant, related to the spectral norms of $\bar{A}_{c d}$ and $\bar{A}_{d s}$, as possible via the choices of $F_{c d}$ and $F_{d c}$ so that the dynamics $\bar{A}$ of the closed-loop extended discrete hybrid system be a convergent matrix. On the other hand, one can choose the $p$-matrix $G_{d}$ of sufficiently small nonnegative entries so that $\bar{B}$ and $\bar{C}$, and then $\bar{S}$ in the second constraint of the Discrete Positive Real Lemma has a sufficiently small spectral norm related to that of $A$ while $\bar{D}+\bar{D}^{T}$ is dominant norm of order $o\left(\left\|G_{d}\right\|_{2}\right)$ over that of $\bar{B}^{T} \overline{P B}$, of order $o\left(\left\|G_{d}\right\|_{2}^{2}\right)$, so that $\bar{R} \succeq 0$ and $\bar{Q} \geq 0$. In this way, the discrete modified closed-loop transfer matrix of $\mathrm{DH}_{c l}$, related to the new input $r(t)$, might be designed to be at least positive real. On the other hand, the asymptotic hyperstability in the mean of $H_{c \ell}$ follows from Theorem 21 from the asymptotic hyperstability of $\mathrm{DH}_{c \ell}$ and the assumptions (2) and (3) of Theorem 21 since the first assumption of such a theorem holds since the controllability of the pair $\left(A_{c}, B_{c}\right)$ implies that of the pair $\left(\bar{A}_{c}, \bar{B}_{c}\right)$.

\section{Conclusions}

This paper has investigated a class of hybrid systems dealt with and characterized with explicit results its positivity and some of its stability properties. The hybrid system consists of a dynamic system which has a continuous-time substate and a digital one with mutual coupled dynamics. An extended discrete hybrid system which describes any hybrid system in the given class at sampling instants is investigated to establish the stability and controllability properties of the discretized system. The state of the extended discrete hybrid system contains the discretized substate of the continuous-time subsystem at sampling instants and the digital substate. The paper studies the stability and controllability, in a robustness context for parametrical disturbance, of such an extended discrete system whose state is defined by both the digital substate and the discretized version of the continuous-time subsystem at sampling instants. Two discrete versions of the KYPLemma are given for (a) a simplified version of the hybrid system related to the relevant pairs of the system and control matrices and (b) for a more general version of such a lemma related to the whole state-space realization involving the output and input-output interconnection matrices as well. The relationships of the positive realness of the transfer matrix to the state-space realization are explicitly characterized related to the discrete KYP-Lemma and Youla's factorization Lemma. The obtained results on positive realness are related to the hyperstability and asymptotic hyperstability properties of the hybrid system for any member of a class of nonlinear and perhaps time-varying controller device satisfying Popov'stype inequality. Finally, some extensions are given for the case where there is a supplementary stabilizing linear control scheme which stabilizes the dynamics hybrid system prior to the nonlinear and time-varying control law operation to establish the hyperstability of the closed-loop system.

\section{Conflicts of Interest}

There are no conflicts of interest related to this paper.

\section{Acknowledgments}

The author is very grateful to the Spanish Government and European Fund of Regional Development FEDER for Grant DPI2015-64766-R and to UPV/EHU by its Grant PGC 17733 for research groups.

\section{References}

[1] M. de la Sen, "The reachability and observability of hybrid multirate sampling linear systems," Computers \& Mathematics with Applications. An International Journal, vol. 31, no. 1, pp. 109-122, 1996.

[2] T. Kaczorek, Positive $1 D$ and $2 D$ Systems, E. D. Sontag and M. Thoma, Eds., Communications and Control Engineering, Springer-Verlag, Berlin, Germany, 2001.

[3] L. Farina and S. Rinaldi, Positive Linear Systems: Theory and Applications, Wiley-Interscience, New York, NY, USA, 2000.

[4] R. Bru, C. Coll, and E. Sanchez, "Structural properties of positive linear time-invariant difference-algebraic equations," Linear Algebra and its Applications, vol. 349, pp. 1-10, 2002.

[5] M. de la Sen, "On positivity and stability of a class of time-delay systems," Nonlinear Analysis: Real World Applications, vol. 8, no. 3, pp. 749-768, 2007.

[6] M. Ait Rami and D. Napp, "Discrete-time positive periodic systems with state and control constraints," Institute of Electrical and Electronics Engineers Transactions on Automatic Control, vol. 61, no. 1, pp. 234-239, 2016.

[7] M. De la Sen, "About the positivity of a class of hybrid dynamic linear systems," Applied Mathematics and Computation, vol. 189, no. 1, pp. 852-868, 2007.

[8] A. Rantzer, "On the Kalman-Yakubovich-Popov lemma for positive systems," Institute of Electrical and Electronics Engineers Transactions on Automatic Control, vol. 61, no. 5, pp. 1346-1349, 2016.

[9] F. Najson, "On the Kalman-Yakubovich-Popov lemma for discrete-time positive linear systems: a novel simple proof and some related results," International Journal of Control, vol. 86, no. 10, pp. 1813-1823, 2013. 
[10] T. Tanaka and C. Langbort, "The bounded real lemma for internally positive systems and $\mathrm{H}$-infinity structured static state feedback," Institute of Electrical and Electronics Engineers Transactions on Automatic Control, vol. 56, no. 9, pp. 2218-2223, 2011.

[11] M. De la Sen, "A result on the hyperstability of a class of hybrid dynamic systems," Institute of Electrical and Electronics Engineers Transactions on Automatic Control, vol. 42, no. 9, pp. 1335-1339, 1997.

[12] M. de la Sen, "Stability of composite systems with an asymptotically hyperstable subsystem," International Journal of Control, vol. 44, no. 6, pp. 1769-1775, 1986.

[13] I. D. Landau, Adaptive Control: The Model Reference Approach, Marcel Dekker, New York, NY, USA, 1979.

[14] W. P. Heath, J. Carrasco, and M. De La Sen, "Second-order counterexamples to the discrete-time Kalman conjecture," Automatica, vol. 60, article no. 6464, pp. 140-144, 2015.

[15] V.-M. Popov, Hyperstability of Control Systems, Springer, New York, NY, USA, 1973.

[16] V. M. Marchenko, "Observability of hybrid discrete-continuous systems," Journal of Differential Equations, vol. 49, no. 11, pp. 1389-1404, 2013.

[17] V. M. Marchenko, "Hybrid discrete-continuous systems: I. Stability and stabilizability," Journal of Differential Equations, vol. 48, no. 12, pp. 1623-1638, 2012.

[18] G. Rajchakit, T. Rojsiraphisal, and M. Rajchakit, "Robust stability and stabilization of uncertain switched discrete-time systems," Advances in Difference Equations, vol. 2012, article no. 134, 2012.

[19] R. Lozano, B. Brogliato, O. Egeland, and B. Maschke, Dissipative Systems Analysis and Control: Theory and Applications, Springer, London, UK, 2nd edition, 2007.

[20] M. Syed Ali and J. Yogambigai, "Synchronization of complex dynamical networks with hybrid coupling delays on time scales by handling multitude Kronecker product terms," Applied Mathematics and Computation, vol. 291, pp. 244-258, 2016.

[21] X. Liu and P. Stechlinski, "Hybrid stabilization and synchronization of nonlinear systems with unbounded delays," Applied Mathematics and Computation, vol. 280, pp. 140-161, 2016.

[22] L.-G. Yuan and Q.-G. Yang, "Bifurcation, invariant curve and hybrid control in a discrete-time predator-prey system," Applied Mathematical Modelling, vol. 39, no. 8, pp. 2345-2362, 2015.

[23] B. Li, "Pinning adaptive hybrid synchronization of two general complex dynamical networks with mixed coupling," Applied Mathematical Modelling, vol. 40, no. 4, pp. 2983-2998, 2016.

[24] Y. Yin, G. Zong, and X. Zhao, "Improved stability criteria for switched positive linear systems with average dwell time switching," Journal of The Franklin Institute, vol. 354, no. 8, pp. 3472-3484, 2017.

[25] X. Zhao, L. Zhang, and P. Shi, "Stability of a class of switched positive linear time-delay systems," International Journal of Robust and Nonlinear Control, vol. 23, no. 5, pp. 578-589, 2013.

[26] L. Wu and J. Lam, "Sliding mode control of switched hybrid systems with time-varying delay," International Journal of Adaptive Control and Signal Processing, vol. 22, no. 10, pp. 909-931, 2008.

[27] E. Kaslik and S. Sivasundaram, "Multistability in impulsive hybrid Hopfield neural networks with distributed delays," Nonlinear Analysis: Real World Applications, vol. 12, no. 3, pp. 16401649, 2011.

[28] E. Beretta, V. Kolmanovskii, and L. Shaikhet, "Stability of epidemic model with time delays influenced by stochastic perturbations," Mathematics and Computers in Simulation, vol. 45, no. 3-4, pp. 269-277, 1998.

[29] M. De La Sen and A. Ibeas, "Stability results for switched linear systems with constant discrete delays," Mathematical Problems in Engineering, vol. 2008, Article ID 543145, 2008.

[30] M. Alberdi, M. Amundarain, A. J. Garrido, I. Garrido, O. Casquero, and M. de la Sen, "Complementary control of oscillating water column-based wave energy conversion plants to improve the instantaneous power output," IEEE Transactions on Energy Conversion, vol. 26, no. 4, pp. 1021-1032, 2011.

[31] P. T. Kabamba and S. Hara, "Worst-case analysis and design of sampled-data control systems," Institute of Electrical and Electronics Engineers Transactions on Automatic Control, vol. 38, no. 9, pp. 1337-1357, 1993.

[32] V. M. Marchenko, "Controllability and observability of hybrid discrete-continuous systems in the simplest function classes," Journal of Differential Equations, vol. 51, no. 11, pp. 1461-1475, 2015.

[33] M. De la Sen, “The generalized Beverton-Holt equation and the control of populations," Applied Mathematical Modelling, vol. 32, no. 11, pp. 2312-2328, 2008.

[34] Z. Bai and H. Lu, "Positive solutions for boundary value problem of nonlinear fractional differential equation," Journal of Mathematical Analysis and Applications, vol. 311, no. 2, pp. 495-505, 2005.

[35] L. H. Erbe and H. Wang, "On the existence of positive solutions of ordinary differential equations," Proceedings of the American Mathematical Society, vol. 120, no. 3, pp. 743-748, 1994.

[36] T. Kailath, Linear Systems, Prentice-Hall, Englewood Cliffs, NJ, USA, 1980.

[37] J. M. Ortega, Numerical Analysis, Academic Press, 1972.

[38] J. H. Taylor, "Strictly Positive-Real Functions and the LefschetzKalman-Yakubovich (LKY) Lemma," IEEE Transactions on Circuits and Systems II: Express Briefs, vol. 21, no. 2, pp. 310-311, 1974. 




Advances in

Operations Research

vatersals

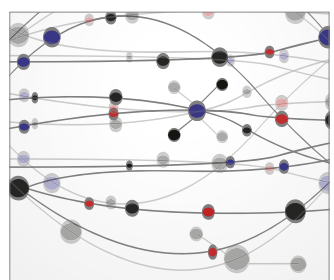

\section{The Scientific} World Journal
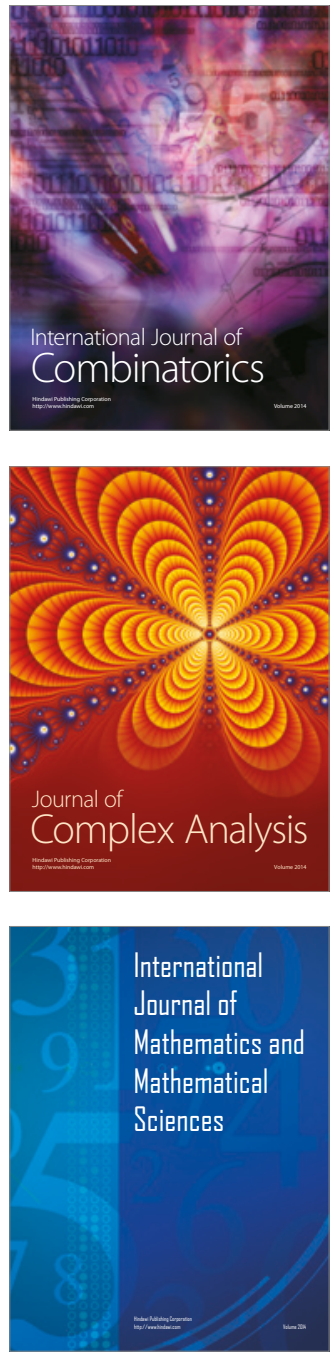
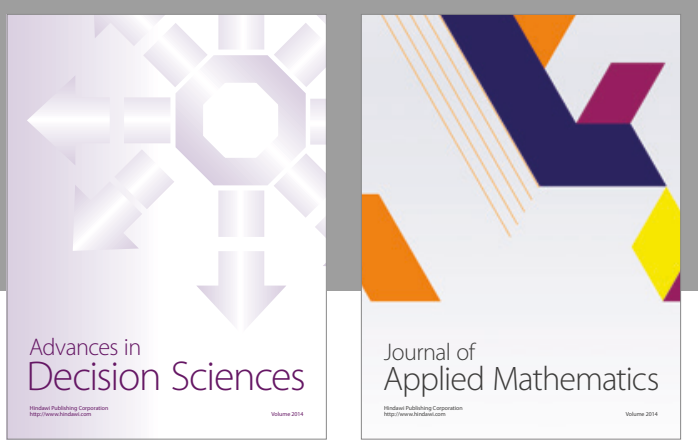

Algebra

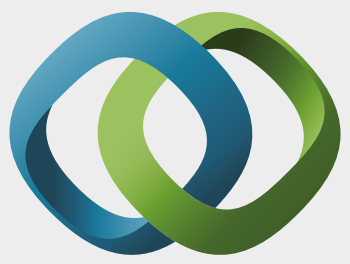

\section{Hindawi}

Submit your manuscripts at

https://www.hindawi.com


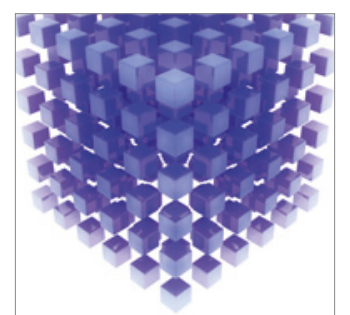

Mathematical Problems in Engineering
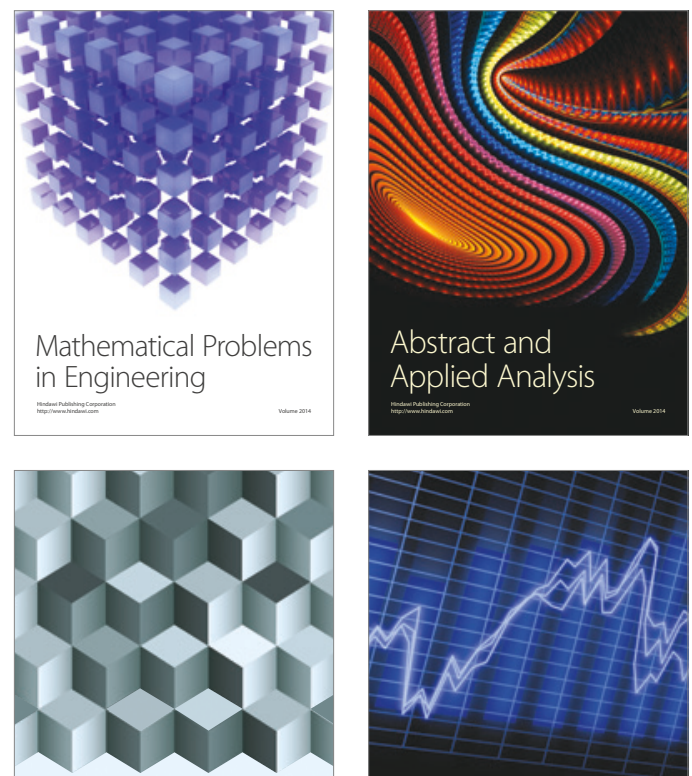

Journal of

Function Spaces



Probability and Statistics
